US WURK LXX (2021), p. 50

\title{
[1530] Das regelmäßige schwache Verb im Mooringer Friesisch: Zwei Konjugationsklassen oder phonologisch bedingte Allomorphie?
}

Nina Hansen, Jarich Hoekstra, Nobuharu Kakuchi, Kim Lilienthal \& Britta Reifferscheidt

In this article we investigate the historical development of the regular weak verb in Mooring, the most vital Mainland North Frisian dialect. We show that until the first half of the 20th century Mooring still distinguished between the two weak conjugation classes inherited from Old Frisian and typical for most Frisian dialects: Class I without and class II with a theme vowel -e- in the endings of the 2nd and 3rd Person Singular Present, the Past and the Past Participle. From the end of the 19th century onward, a process of deletion of schwa after sonorants gradually caused the fusion of class II weak verbs with a stem-final vowel or sonorant with class I weak verbs. After World War II this process came to its (near) completion and the former morphological division of the weak verbs in two conjugation classes was given up in favour of a phonological distribution of the endings on the basis of the stem-final segment: Endings with e appear after obstruents, endings without e after vowels and sonorants. Although modern grammars in principle recognized this new phonological conditioning of the weak conjugation, they failed to see that there remained a number of exceptions, viz. former class I weak verbs with a stem-final obstruent still taking an eless ending. That one is dealing with exceptions here is clearly shown, however, by the fact that these verbs gradually adapt to the phonological conditioning and assume endings with e in modern Mooring.

\section{Einleitung}

Wer sich als SprachlernerIn daran macht, die Feinheiten der Verbmorphologie des Mooringer Friesisch zu ergründen, sieht sich vor mehrere Hürden gestellt. ${ }^{1}$ Nicht allein hat das Mooring eine beträchtliche Zahl von Verben,

1. Dieser Aufsatz ist der Niederschlag eines Forschungsprojekts zum schwachen Verb im Mooringer Friesisch, das im Rahmen des Thematischen Hauptseminars Sprachwissenschaft im Fach Frisistik an der Christian-Albrechts-Universität zu Kiel im Us Wurk 70 (2021), s. 50-85; https://doi.org/10.21827/5fb7c8ffe4618 


\section{US WURK LXX (2021), p. 51}

die Irregularitäten im Verbstamm aufweisen (vgl. Köbernik 2014), auch die Regeln für die Konjugation des regelmäßigen schwachen Verbs, wie sie in Grammatiken und Lehrbüchern aufgeführt werden, sind alles andere als eindeutig. Wer z. B. der tonangebenden neueren Grammatik des Mooring von Tams Jörgensen (1972, 27-28) entnommen hat, dass es schwache Verben mit und ohne $e$ in der Präteritumendung $(-(e) d)$ gibt und dass dieses $e$ fehlt bei Verben, die (im Infinitiv) auf -le, -me, -ne, -re und -we oder auf Diphthong $+e$ enden, wird schnell feststellen, dass diese Beschreibung den Tatsachen nur zum Teil gerecht wird. Erstens gibt es wahrscheinlich mehr Verben mit einem Stamm auf $-w$, die ein $e$ aufweisen (hoowe 'hoffen' hoowed 'hoffte'), als solche, bei denen dies nicht auftritt (täiwe 'warten' täiwd) und zweitens fehlt das $e$ auch bei vielen Verben, die nicht zur besagten Gruppe gehören: Das $e$ wird nicht nur bei Verben auf die Sonoranten $l, r, m$ und $n$ weggelassen, sondern auch bei solchen auf $n g$ ( $f a ̊ n g e$ 'fangen' - fångd 'fing'), nicht nur bei Verben auf einen Diphthong, sondern auch bei solchen auf einen kurzen oder langen Monophthong (fülie 'folgen' - fülid 'folgte', bü̈̈e 'arbeiten [auf dem Feld]' - bü̈̈d 'arbeitete'), und sogar bei einer Reihe von Verben, die auf einen Obstruenten enden, tritt das $e$ nicht auf (brüke 'gebrauchen' - brükd 'gebrauchte', klape 'schneiden [mit der Schere]' - klapd 'schnitt').

Die lückenhafte und verwirrende Darstellung der Konjugation des schwachen Verbs im Mooring bei Jörgensen (1972) und darauf basierenden Grammatiken und Lehrbüchern kann, abgesehen von eher zufälligen Ungenauigkeiten in der Beschreibung, mehrere Gründe haben. Möglicherweise haben die VerfasserInnen dieser Werke die schwache Konjugation im Mooring nicht richtig einordnen können, weil sie diese zu sehr vom Standpunkt der benachbarten Hochsprachen Deutsch und Dänisch mit ihrer einheitlichen schwachen Konjugation und zu wenig aus der Perspektive der Entwicklung der traditionellen zwei schwachen Konjugationsklassen im Friesischen betrachtet haben. Die Entwicklung der zwei schwachen Klassen, gerade in denjenigen Mundarten, in denen die traditionelle Zweiteilung unter Druck steht oder bereits verschwunden ist (den nördlichen nordfriesischen Festlandmundarten und dem Wangeroogischen), wurde in letzter

Sommersemester 2020 durchgeführt wurde. Für hilfreiche Kommentare zu einer früheren Fassung dieses Textes danken wir Temmo Bosse, Lena Terhart, Wendy Vanselow und Alastair Walker. Ein besonders großer Dank geht an Nils Århammar, der uns seinen Vortrag (Århammar 1995) und seine Materialien zur schwachen Verbkonjugation im Festlandnordfriesischen zur Verfügung stellte, und uns auch sonst mit nützlichen Hinweisen unterstuitzt hat. 


\section{US WURK LXX (2021), p. 52}

Zeit von Temmo Bosse in einer Reihe von Arbeiten stärker ins Blickfeld gerückt (Bosse 2012, 2013, 2019, Kap. 4). Es könnte auch sein, dass die schwache Konjugation im (neueren) Mooring so stark im Wandel begriffen ist und derartigen Schwankungen im Gebrauch unterliegt, dass es schwer ist, überhaupt feste Regeln dafür zu formulieren. Eine genauere Untersuchung der diachronen Entwicklung und des synchronen Status des schwachen Verbs im Mooringer Friesisch scheint auf jeden Fall geboten zu sein.

In diesem Aufsatz werden wir zunächst (in Abschnitt 2) einen ersten tentativen Überblick über die Konjugation des schwachen Verbs im heutigen Mooring geben. Bei der Beschreibung kristallisieren sich einige Problemkomplexe heraus, die in den nachfolgenden Abschnitten weiter untersucht werden sollen. Zentral dabei scheint die Frage zu sein: Gibt (gab) es im Mooringer Friesisch noch die traditionelle Einteilung in Verben der 1. und der 2. schwachen Konjugationsklasse oder ist diese durch eine phonologisch bedingte Verteilung der Endungen auf Grund des Stammauslauts ersetzt worden, wie es die Darstellung bei Jörgensen (1972) im Prinzip nahelegt? Zuerst gehen wir (in Abschnitt 3) der Frage nach, wie die Konjugation des schwachen Verbs im Laufe der Zeit in den Grammatiken und grammatischen Abrissen des Mooring beschrieben worden ist. Anschließend (in Abschnitt 4) diskutieren wir kurz das Phänomen der Tilgung von Schwa nach Sonoranten, das sich in der Geschichte des Mooringer Friesisch manifestiert hat. Danach untersuchen wir (in Abschnitt 5), ob sich gewisse Änderungen in der Konjugation des schwachen Verbs in der mooringerfriesischen Textüberlieferung nachweisen lassen. Schließlich werden die Ergebnisse in einem Fazit (Abschnitt 6) zusammengefasst. ${ }^{2}$

\section{Ein erster Überblick}

Das Friesische verfügt von alters her über zwei Klassen von regelmäßigen (schwachen) Verben, die auf die $a$ - und $i a$-Verben im Altfriesischen zurückgehen (Bremmer 2009, 78-80, 85). In den meisten friesischen Mundarten sind diese zwei Konjugationsklassen erhalten geblieben. Im Wangeroogischen scheinen sie aber zu Gunsten einer phonologisch bedingten

2. Unsere Ergebnisse wurden zum Teil schon in einem Vortrag von Nils Århammar für die AG Sprache und Literatur des Nordfriisk Instituuts aus dem Jahr 1995 vorweggenommen. Dieser Vortrag blieb leider unpubliziert, aber Århammar gebührt die Ehre, als Erster den historischen Umbruch im System der schwachen Verben im Mooring und in den anderen nördlichen Festlandsmundarten erkannt zu haben (vgl. auch Århammar 2001, 756). 


\section{US WURK LXX (2021), p. 53}

Verteilung der Endungen aufgegeben worden zu sein (Bosse 2012). Auch in den nördlichen nordfriesischen Festlandmundarten ist ihr Status nicht besonders klar und man könnte tendenziell eine ähnliche Entwicklung wie im Wangeroogischen vermuten (Århammar 2001, 756, Hoekstra 2001, 778). Im Mooring ist der Unterschied zwischen den zwei Klassen für SprachwissenschaftlerInnen noch teilweise zu erkennen, aber möglicherweise nicht mehr so ausgeprägt, dass SprachlernerInnen ihn noch als solchen identifizieren könnten. Die nachfolgende Beschreibung basiert weitgehend auf Hoekstra (2015).

Im Mooringer Friesisch höbe sich die 2. schwache Klasse von der 1. schwachen Klasse nur noch dadurch ab, dass in der 2. und 3. Person Singular Präsens, im ganzen Präteritum und im Partizip Perfekt zwischen Stamm und Personalendung ein Themavokal - $e$ - erscheint; die Personalendungen wie auch die Präteritum- und Partizip-Perfekt-Endung (das Dentalsuffix $d)^{3}$ wären in beiden Klassen gleich. Vergleiche dazu die Paradigmen der Verben teele und frååge:

(1) Klasse I:

INFINITIV I: teel|e 'zählen; rufen' (afr. tella)

INFINITIV II: teel|en

$\begin{array}{llll}1 \text { SINGULAR } & \text { PRÄSENS } & \text { PRÄTERITUM } & \text { PARTIZIP } \\ 2 & \text { teel } \mid \varnothing & \text { teel }|d| \varnothing & \text { teel } \mid d \\ 3 & \text { teel } \mid \text { st } & \text { teel }|d| \text { st } & \\ 123 \text { PLURAL } & \text { teel } \mid t & \text { teel }|d| \varnothing & \\ \text { teel } \mid e & \text { teel }|d| e n & \text { IMPERATIV teel } \mid \varnothing\end{array}$

Klasse II:

INFINITIV I: frååg|e 'fragen' (afr. frāgia)

INFINITIV II: frååg|en

PRÄSENS PRÄTERITUM PARTIZIP

1 SINGULAR frååg $\mid \varnothing \quad$ frååg $|e| d \mid \varnothing \quad$ frååg $|e| d$

$2 \quad$ fråag $|e| s t \quad$ frååg $|e| t \mid s t$

$3 \quad$ frååg $|e| t \quad$ frååg $|e| d \mid \varnothing$

123 PLURAL frååg $\mid e \quad$ frååg $|e| d \mid e n \quad$ IMPERATIV frååg|Ø

3. Das Dentalsuffix <d> wird nach stimmhaften Konsonanten und betontem Vokal als [d], nach stimmlosen Konsonanten und nach Schwa (+ Sonorant) als [t] realisiert (vgl. Walker \& Wilts 2001, 297). In teeld 'rief' und skraid 'weinte' wird demnach ein [d], in brükd 'gebrauchte', frååged 'fragte' und soomeld 'sammelte' ein [t] gesprochen. 


\section{US WURK LXX (2021), p. 54}

as Mooring hatte den charakteristischen Themavokal $-i$ - im Infinitiv, in der 1. Person Singular Präsens, im Präsens Plural und im Imperativ der 2. Klasse schon vor der Überlieferung aufgegeben, so dass die 2. Klasse in diesen Formen nicht mehr von der 1 . Klasse zu unterscheiden ist. ${ }^{4}$

Noch stärker verwischt ist die Opposition zwischen den beiden Klassen aber dadurch, dass Verben der 2. Klasse, die auf Vokal oder Sonorant $(l, r$, $m, n, n g$ ) enden, das thematische - $e$ - weglassen und somit genauso konjugiert werden wie Verben der 1. Klasse. Vergleiche dazu das Paradigma des Verbs hååle:

(2) Klasse II:

INFINITIV I: håål|e 'holen' (afr. halia)

INFINITIV II: håål|en

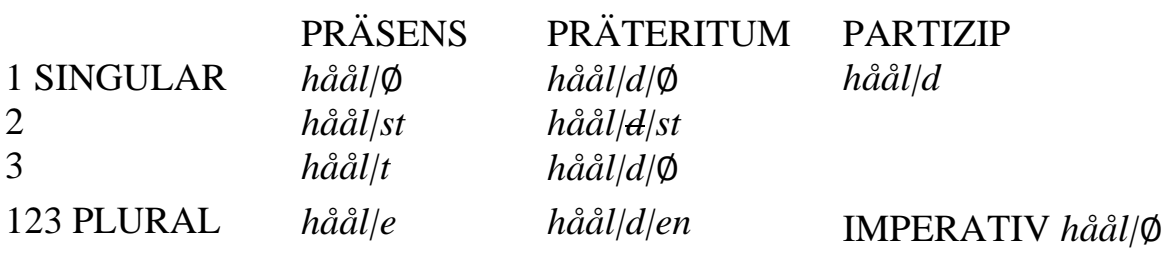

So auch: seenie 'sündigen' (afr. sendigia), spååre 'sparen' (afr. sparia), tiine 'dienen' (afr. thiānia), schoome '(sich) schämen' (afr. skomia), fånge 'fangen' (zu diesem Verb siehe unten Abschnitt 5)

Wie wir im folgenden Abschnitt feststellen werden, hatte sich die Tilgung von Schwa nach Sonoranten bei Bende Bendsen (1. Hälfte des 19. Jh.) noch nicht vollzogen, so dass sich die Frage stellt, wann diese sich durchgesetzt hat und welche Konsequenzen dies für die Analyse der schwachen Konjugation hat.

Bei Verben mit einem Stamm auf Konsonant + Sonorant, die historisch alle zur 2. schwachen Klasse gehören, war bereits am Anfang der Überlieferung (z. B. bei Bende Bendsen) der Themavokal - $e$ - weggefallen, so dass diese schon früh mit den Verben der 1. schwachen Klasse zusammengefallen waren. Zwischen den beiden finalen Stammkonsonanten wird

4. Im Süder- und Mittelgoesharder Friesisch ist (war) der Themavokal - $i$ - noch vorhanden (vgl. sgo., mgo. freegi 'fragen'). Interessant in diesem Zusammenhang ist das Nordergoesharder Friesisch, wo das - $i$ - im 18. Jahrhundert noch in Erscheinung tritt (vgl. Boy Jacobsen: fregiä 'fragen'), im 19. Jahrhundert aber restlos verschwunden ist (vgl. Bosse 2013). 


\section{US WURK LXX (2021), p. 55}

bei diesen Verben ein Schwa eingefügt, wenn eine konsonantische Endung $(-s t,-t,-d)$ oder keine Endung folgt: ${ }^{5}$

(3) INFINITIV

$$
\text { 1.SG.PRÄS/ 3.SG. PRÄT/ }
$$

IMP PRÄS PART

\begin{tabular}{|c|c|c|c|c|}
\hline soomle & 'sammeln' & soomel & - $\quad$ soomelt & - soomeld \\
\hline hoonle & 'handeln’ & hoonel & - hoonelt & hooneld \\
\hline daingle & 'schlenkern' & daingel & - daingelt & dangeld \\
\hline bü̈̈ntjle & ‘wickeln’ & büüntjel & büntjelt & büüntjeld \\
\hline boolne & 'entzünden' & boolen & - boolent & boolend \\
\hline duulme & ‘dösen’ & duulem & - duulemt & - duulemd \\
\hline bloosme & ‘blühen’ & bloosem & - bloosemt & - bloosemd \\
\hline tiikne & 'zeichnen' & tiiken & tiikent & - tiikend \\
\hline räägne & 'rechnen' & räägen & räägent & räägend \\
\hline sikne & 'seufzen' & siken & - sikent & - sikend \\
\hline krasne & 'taufen’ & krasen & - krasent & - krasend \\
\hline
\end{tabular}

Bei Verben mit einem Stamm auf $r+$ Sonorant findet aber keine SchwaEpenthese statt:

(4)

$$
\begin{array}{llllllll}
\text { stiirme } & \text { 'riechen' } & - & \text { stiirmt } & - & \text { stiirmd } & \text { - } & \text { stiirmd } \\
\text { scharne } & \text { 'buttern' } & - & \text { scharnt } & - & \text { scharnd } & - & \text { scharnd } \\
\text { kjarle } & \text { 'gerinnen' } & - & \text { kjarlt } & - & \text { kjarld } & - & \text { kjarld }
\end{array}
$$

5. Bei den Verben mit einem Stamm auf Konsonant + Sonorant handelt es sich konkreter um Verben auf Konsonant + Nasal und Verben auf Sonorant $+l$ (außer denen auf $-r l$ ). Verben, die auf Konsonant $+r$ enden, sind nicht vorhanden; bei Verben, die hier theoretisch in Betracht kämen (vgl. moor. bä̈̈were - bääwert mit fa. beewre - beewert) ist das $e$ zwischen Konsonant und $r$ im Mooring nicht epenthetisch, sondern fester Bestandteil des Stammes:

$$
\begin{aligned}
& \text { (i) bääwere 'beben' } \\
& \text { ståmere 'stottern' } \\
& \text { slåådere 'tratschen' } \\
& \text { bulere 'poltern' }
\end{aligned}
$$

Auch Verben auf Nicht-Sonorant $+l$ kommen nicht vor:

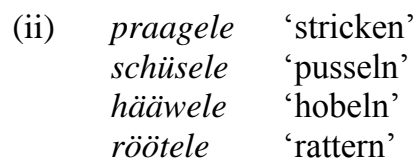

Bei Verben auf Sonorant $+l$ gibt es übrigens Variation; neben soomle 'sammeln' steht z. B. schamele 'schimmeln'. 


\section{US WURK LXX (2021), p. 56}

In der ganzen mooringerfriesischen Überlieferung findet man bei Verben auf Konsonant + Sonorant neben Endungen ohne $e$ (mit Schwa-Epenthese) wie in (3) auch solche mit $-e$ (ohne Schwa-Epenthese). Schon Bendsen schwankt zwischen beiden Möglichkeiten: bōllent oder bōllnet, geschworen [= entzündet] (BB 198), Dāt Bjàrn ás noch ài krástent oder krástnet, das Kind ist noch nicht gechristnet, d.i. getauft (BB 417). So auch in späteren Texten liknet statt likent, bloosmet statt bloosemt, soomlet statt soomelt usw. Solche Fälle weisen wahrscheinlich auf das Verschwinden der Epentheseregel hin (Verben auf Konsonant + Sonorant erhalten statt der Endung $-t$ und Schwa-Epenthese die Endung - et, um das unerlaubte Auslautcluster zu vermeiden), das Phänomen müsste aber näher untersucht werden. Wir lassen diese Variation in unserer Untersuchung weiter außer Acht. ${ }^{6}$

Eine Opposition zwischen Verben der 1. und 2. schwachen Klasse gäbe es somit im heutigen Mooring nur noch bei Verben mit einem Stamm auf Obstruent. Vergleiche folgende 'Minimalpaare' (mit der 3. Person Singular Präsens): ${ }^{7}$

(5) 1. schwache Klasse

2. schwache Klasse

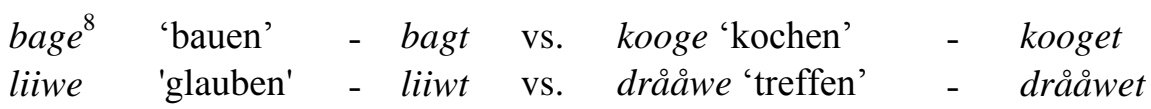

Wie liiwe auch: täiwe 'warten', kliiwe '(Holz) spalten', schuurwe 'zerschneiden, ${ }^{9}$

liise 'lösen’ - liist vs. pååse 'passen' - pååset

Wie liise auch: räise 'reisen', riise 'errichten; erheben'

6. Eine alte Schwa-Epenthese zwischen Liquiden $(l, r)$ und $w$ zeigen z. B. die unregelmäßigen Verben scheelwe 'zittern' - scheelewt 'zittert' und iirwe 'erben' - årewt 'erbt'. Für scheelewt (so Jörgensen 1972) findet man auch scheelwet (so Wilts 1995). Vergleiche auch stärwe 'sterben' - steerewt 'stirbt' (BB 304), heute steerft (Jörgensen 1972, Wilts 1995).

7. Wir verzeichnen hier absichtlich die 'klassischen' (älteren) Formen der Verben der 1. schwachen Klasse und lassen zunächst unberücksichtigt, dass die meisten von ihnen heutzutage auch neue, regelmäßige Formen (mit $e$ ) aufweisen können (siehe aber Abschnitt 5.4. und die Beispiele in (19)).

8. Neben bage (Ostermooring) steht bäge (Westermooring).

9. Die letzten zwei Verben werden im 'Frasch Leseböck' (Ingwersen \& Johannsen 1926, 12) als Verben der 1. schwachen Klasse, d.h. als Verben ohne thematisches - $e$-, aufgeführt. 


\section{US WURK LXX (2021), p. 57}

$$
\begin{aligned}
& \text { seete 'setzen', - seet vs. weete 'wetzen' - weetet } \\
& \text { Wie seete auch: fleete 'umziehen', kneete 'knoten', swate 'schwitzen', } \\
& \text { stjarte 'stürzen', maste 'missen' } \\
& \text { klape 'schneiden' } \quad \text { - klapt } \quad \text { vs. } \quad \text { stoope 'stopfen' - stoopet } \\
& \text { Wie klape auch: kape 'kippen', snåpe 'schnappen', stiipe '(Kerzen) ziehen' } \\
& \text { brüke 'gebrauchen' } \quad \text { brükt vs. } \quad \text { snååke 'reden' } \quad \text { snååket } \\
& \text { Wie brüke auch: mårke 'merken', röke 'rauchen', schake 'schicken', } \\
& \text { reeke 'recken', sleeke 'löschen', streeke 'strecken', teeke 'Dach decken'11 }
\end{aligned}
$$

Die Zahl der (einigermaßen häufig vorkommenden) Verben der 1. Klasse mit einem Stamm auf Obstruent ist aber überschaubar, so dass leicht der Eindruck entstehen könnte, als sei die Verteilung der Endungen im Mooringer Friesisch phonologisch gesteuert: Nach Vokal und Sonorant eine Endung ohne $e$, nach Obstruent eine Endung mit $e$. Die in der Einleitung angeführte Grammatik von Tams Jörgensen (1972) scheint ebendies nahelegen zu wollen.

Soweit wir sehen, gibt es jetzt für das heutige Mooring deshalb zwei mögliche synchrone Analysen:

- Es gibt noch zwei schwache Konjugationsklassen, eine ohne und eine mit Themavokal -e-; der Unterschied zwischen beiden Klassen ist aber bei Verben auf Sonorant neutralisiert, so dass er nur noch bei Verben auf Obstruent zu Tage tritt.

- Es gibt beim schwachen Verb eine phonologische Verteilung der Endungen - $(e) s t /-(e) t$ und $-(e) d$ - (in denen der ehemalige Themavokal $-e$ - als Teil der Personalendung bzw. der Präteritum- und Partizip-PerfektEndung reanalysiert ist) ${ }^{12}$ auf Grund des Stammauslauts, aber mit einer

10. Bei Verben mit einem Stamm auf $-t$ ist die Konsonantenfolge $t t(\mathrm{z}$. B. in $/ \mathrm{seet} /+\mathrm{t} /$ ) automatisch zu $t$ vereinfacht.

11. Bendsen $(1860,300)$ verzeichnet rêke (rêkt - riggd - riggd), slêke (slêkt - sliggdsliggd), strêke (strêkt - striggd - striggd) und têke (têkt - tiggd - tiggd) mit Stammallomorphie (vgl. auch Bauer 1925, 101), gibt aber an, dass diese Verben "auch ohne Anstoss regelmässig abgewandelt werden (können)". Nis Albrecht Johannsen schreibt reekt/reekd (aber für das transitive 'recken' auch reeket) und streekt/streekd, Emil Hansen hat teekd.

12. Wir werden im Folgenden meistens bequemlichkeitshalber von der Endung - $(e t)$ usw. oder von Endungen mit oder ohne $e$ sprechen, unabhängig davon, ob das $e$ noch ein Themavokal ist oder schon Teil der Personalendung bzw. der Präteritum- und PartizipPerfekt-Endung geworden ist. 


\section{US WURK LXX (2021), p. 58}

Restgruppe von Ausnahmen (die historisch auf Verben der 1. schwachen Klasse mit Stamm auf Obstruent zurückgehen).

Im Falle der ersten Analyse mutet es vielleicht merkwürdig an, dass der Bereich der zwei Konjugationsklassen phonologisch eingeschränkt wird. Kann man noch von zwei Konjugationsklassen sprechen, wenn der Unterschied nur bei einem Teil der schwachen Verben (denen auf Obstruent) zu Tage tritt? Im Falle der zweiten Analyse ist die Frage, wie man mit den Ausnahmen in (5) verfährt. Sie müssten als unregelmäßig gelistet werden und es wäre in so einem Fall auch zu erwarten, dass sich insbesondere die weniger häufig vorkommenden unter ihnen nach und nach der allgemeinen phonologischen Verteilung beugen würden (d.h. eine Endung mit $e$ annehmen würden) und dass ihre Zahl abnehmen würde. Dies gilt es im Folgenden zu prüfen.

\section{Das schwache Verb in den Grammatiken und Lehrbüchern}

Wenn man sich die Grammatiken und Lehrbücher des Mooring vornimmt und sie auf die Behandlung des schwachen Verbs hin untersucht, zeichnet sich zeitlich und inhaltlich eine klare Zweiteilung ab: Bis in die Zwischenkriegszeit bleibt Bende Bendsen (1860) das Vorbild, nach dem Zweiten Weltkrieg orientiert man sich an Tams Jörgensen (1972). Während man bei Tams Jörgensen grundsätzlich eine phonologisch konditionierte Verteilung der Endungen findet (die, wie wir festgestellt haben, einige Fragen offen lässt), lassen sich in der Beschreibung von Bende Bendsen, obwohl er diese nicht explizit unterscheidet, die zwei traditionellen schwachen Konjugationsklassen noch klar erkennen.

Bende Bendsen geht in seinem Buch 'Die Nordfriesische Sprache nach der Moringer Mundart' (1860, 295-296) davon aus, dass regelmäßige schwache Verben im Mooringer Friesisch eine Endung mit $e$ erhalten: ${ }^{13}$

Das ganz regelmässige Zeitwort endigt sich in der gewesenen Gegenwart (Imperfectum) und in der Vergangenheit (Perfectum) allemal auf -et. (S. 295)

Nur regelmäßige Verben, die auf Konsonant + Sonorant oder auf -ig (heute $-i$ ) enden, bekommen bei ihm eine Endung ohne $e$, dafür aber SchwaEpenthese im stammfinalen Konsonant + Sonorant-Cluster (S. 296), dies

13. Obwohl Bendsens Buch erst 1860 veröffentlicht wurde, geht es auf ein bereits 1830 vorliegendes Manuskript zurück. Eine frühere handschriftliche Fassung seiner Sprachlehre hatte Bendsen schon 1824 fertiggestellt (vgl. Feitsma 1990). 


\section{US WURK LXX (2021), p. 59}

größtenteils in Übereinstimmung mit der Lage im heutigen Mooring (eine Abweichung wird in Abschnitt 5.1. erörtert).

Die Verben, die Bendsen als "ganz regelmäßig" bezeichnet, gehören, historisch gesehen, alle zur 2. schwachen Klasse. Es fällt außerdem auf, dass der Wegfall von $e$ bei Verben der 2. schwachen Klasse mit einem auf Sonorant endenden Stamm bei ihm noch nicht stattgefunden hat. Dies ist ein wichtiger Punkt, weil es gerade dieser Prozess ist, der die herkömmliche Unterscheidung der zwei Konjugationsklassen im Mooringer Friesisch zerrüttet haben könnte.

Alle anderen Verben gelten bei Bende Bendsen (S. 296-299) als unregelmäßig, nicht nur die starken Verben und die schwachen Verben mit Stammallomorphie (alles Verben mit einer Endung ohne $e$ ), sondern auch die schwachen Verben ohne Stammallomorphie, die eine Endung ohne $e$ erhalten, in Bendsens Worten "die am wenigsten unregelmäßigen Zeitwörter", wie z.B. bággen 'bauen', bággd. Die schwachen Verben, die von Bendsen als unregelmäßig eingestuft werden, sind leicht als die Verben der 1. schwachen Klasse zu identifizieren.

Dies bedeutet in der Zusammenschau, dass bei Bende Bendsen, obwohl er sie selber nicht als solche erkannt hat, die historischen zwei schwachen Konjugationsklassen noch völlig intakt sind. ${ }^{14}$ Bendsen illustriert dies einleuchtend an Hand von folgendem 'Minimalpaar':

fállen, fillen, ick fálld ... Das Wort fállen, einem wie ein Füllen nachlaufen, von dāt Fáll, das Füllen, ist nicht mit fállen, schinden, von dāt Fall (Fell), zu verwechseln; jenes ist regelmässig: he fállet me êfter, er läuft mir nach. (S. 297-298)

Die auf Sonorant endenden Homonyme falle 'Fell abziehen' $(<$ afr. *filla $<$ germ. *felljan) und falle 'wie ein Fohlen nachlaufen' (ein jüngeres denominatives Verb zu fal 'Fohlen') unterscheiden sich dadurch, dass ersteres zur 1. und letzteres zur 2. schwachen Klasse gehört. Im heutigen Mooring würden beide Verben gleich (ohne $e$ ) konjugiert werden.

Die Behandlung der zwei Klassen von schwachen Verben bei Bende Bendsen als "ganz regelmäßig" und "am wenigsten unregelmäßig" mag auf den ersten Blick sonderbar erscheinen, sie ist aber gut nachzuvollziehen,

14. Lyngby unterscheidet in seiner kleinen Monographie zum Nordfriesischen in der Böking- und Wiedingharde $(1858,43-44)$ für das Friesische von Naibel/Niebüll unter Verweis auf Rasmus Rasks Grammatik des Altfriesischen (Rask 1825) ebenfalls zwei Klassen von schwachen Verben (Beispiele: hire 'hören' - hird - hird und måge 'machen' - måget-måget). 


\section{US WURK LXX (2021), p. 60}

wenn man Bendsens Grundvoraussetzungen berücksichtigt. Er analysiert das mooringerfriesische Verbsystem aus der Sicht der deutschen (oder dänischen) Grammatik und die Vorstellung, dass es mehr als eine regelmäßige schwache Klasse geben könnte, kam somit für ihn kaum in Betracht. Dass er dann die 2. schwache Klasse als die "ganz regelmäßige" ansetzt, ist eine folgerichtige Entscheidung. Die 2. schwache Klasse ist in friesischen Dialekten, die noch über die beiden Klassen verfügen, die unmarkierte Klasse (vgl. Tiersma 1999², 65, Hoekstra 1998, 141, Dyk 2020); neue Verben, die durch Konversion oder Affigierung von Nicht-Verben abgeleitet werden, gehören zu dieser Klasse. ${ }^{15}$ Im Mooring ist sie außerdem die Klasse, die keine Irregularität im Stamm aufweist. Stammallomorphie tritt im Mooring in der 1. schwachen Klasse auf, so dass man sich über Bendsens Entscheidung, letztere mit den starken Verben als unregelmäßig einzustufen, nicht zu wundern braucht. Bendsen hat aber gesehen, dass es auch Verben der 1. Klasse ohne Stammallomorphie gibt, die bei ihm dann durchaus konsequent als "am wenigsten unregelmäßig" gelten.

Bendsen verzeichnet als normale Endung des Präteritums und des Partizip Perfekts bei den 'regelmäßigen' Verben -et, aber nach seiner Aussage (S. 297) kann es bei einigen Verben Wechsel mit -ed geben (ick lühssed oder lühsset 'ich lauste') und auch bei dem adjektivisch verwendeten flektierten Partizip tritt bei ihm Variation zwischen -et (de rōsete Shájnkel 'der verfaulte Schinken') und -ed (de tōpede Kräjdder 'der gehaubte Hahn') auf (S. 296), wobei er die genaue Erklärung dieser Variation schuldig bleibt. Bei Verben mit einem Stamm auf unbetonte Silbe ist die Endung immer - $t$ (dá sträujnnigte Matrose, 'die gestrandeten Matrosen'). Bei den 'unregelmäßigen' Verben ist die Endung - $d$. Wahrscheinlich stimmte die Aussprache des Dentalsuffixes bei Bendsen im Großen und Ganzen mit der heutigen Aussprache überein (vgl. Fußnote 3).

Erika Bauer (1925, 100-103) betrachtet, ähnlich wie Bendsen, die Endung mit $e$, bei ihr $-e d$, als die normale Präteritumendung bei schwachen Verben (S. 100-101). Auch Verben, die auf Sonorant enden, können bei ihr diese Endung erhalten (S. 101). Bauer bezeichnet alle schwachen Verben, die auf $-d$ enden (mit oder ohne Stammallomorphie), als "Abweichungen von dieser allgemeinen Präterialbildung" (S. 101). Anders als Bendsen nimmt sie also die starken Verben nicht mit in diese letzte Gruppe hinein.

15. Auch entlehnte Verben können in der 2. schwachen Klasse landen (z. B. fa. maile 'mailen'), hier ist die Lage aber, je nach Mundart, ein wenig komplizierter, weil Interferenz mit der Gebersprache dabei eine Rolle spielen kann. 


\section{US WURK LXX (2021), p. 61}

Bauer gibt folgende Erklärung für die Entwicklung der Präteritumendung: Erstens geht sie davon aus, dass die alte Endung der 2. schwachen Klasse, afr. -ade, durch Reduktion mit der Endung der 1. schwachen Klasse -de zusammengefallen ist. Zweitens meint sie, dass die Endung -de der 1. schwachen Klasse zuerst $\mathrm{zu}-d$ geworden ist und dass sich danach im Regelfall nach Plosiven und Frikativen zwischen Stamm und Endung ein Sprossvokal gebildet hat, der später auf Stämme mit auslautendem Sonoranten übertragen wurde.

Bauers historische Analyse der Endung des Präteritums ist schwer nachvollziehbar. In allen friesischen Mundarten ist das $e(<a)$ in der Endung der 2. schwachen Klasse erhalten geblieben. Die Beispiele mit -ed, die sie anführt, betreffen dann auch alle Verben dieser Klasse. Dies wäre ein merkwürdiger Zufall, wenn die Endung -ed durch die von ihr angenommene Schwa-Epenthese entstanden wäre. Obwohl sie die historischen Hintergründe nicht durchschaut hat, zeigt ihre Darstellung aber im Grunde, wie bei Bendsen, die Unterscheidung der beiden Konjugationsklassen.

Das 'Frasch Leseböck' (Ingwersen \& Johannsen 1926) gibt im einleitenden grammatischen Abriss keine Regeln für die Konjugation des schwachen Verbs. Es erläutert diese zwar mit Beispielen (S. 12), erklärt aber nicht, weshalb liewt 'glaubt' neben lowet 'verspricht', beggt 'baut' neben maaget 'macht', und galt 'weint' neben spalet 'spielt' steht. Die aufgeführten Formen der schwachen Verben stimmen jedoch in jeder Hinsicht mit Bende Bendsens Beschreibung überein.

In Tams Jörgensens Grammatik (1972, 27-28) wird zum ersten Mal eine phonologische Verteilung der Endungen vorgeschlagen: Schwache Verben sind bei ihm diejenigen, die im Präteritum eine Endung - $(e) d$ bekommen. Die normale Endung ist die mit $e$ - Jörgensen erläutert dies an Hand des Paradigmas von frååge - und dieses $e$ fällt in der Beugung nach Verben auf $-i e,-l e,-m e,-n e,-r e,-w e$ und auf Diphthong + $e$ weg: $:^{16}$

Bai da uurde, wat aw -ie, -le, -me, -ne, -re än -we iinje, unti weer dåt - $e$ eefter en dööweltlüd fülit, fålt dåt $e$ bait biien wäch. (Bei den Wörtern, die auf $i e,-l e,-m e,-n e,-r e$ und -we enden, oder wo das - $e$ hinter einem Diphthong folgt, fällt das $e$ bei der Beugung weg.)

16. Aus historischer Perspektive ist diese Beschreibung ein wenig irreführend. Viele Verben mit einer Endung ohne $e$, die Jörgensen als Beispiele anführt (teele 'rufen', kåne 'kennen', hiire 'hören'), ursprünglich Verben der 1. schwachen Klasse, hatten schon im Altfriesischen kein $e$. Es fällt hier also historisch gesehen nichts weg. 


\section{US WURK LXX (2021), p. 62}

Wenn man Verben mit einem Stamm auf $n g$ und Monophthong dazu zählen darf, könnte man dies wie folgt zusammenfassen: nach Verben mit einem Stamm auf Vokal oder Sonorant steht eine Endung ohne $e$. Nur -we passt hier nicht richtig ins Bild und ist, wie schon in der Einleitung angedeutet, problematisch. Jörgensen führt zwar das Beispiel täiwe 'warten' (täiwt täiwd) auf, lässt aber die Verben mit einem Stamm auf $-w$, die ein $e$ in der Endung haben, wie z. B. hoowe 'hoffen' (hoowet - hoowed), unerwähnt.

Die heutige Schreibweise der Präteritum- und Partizip-Perfekt-Endung als -(e)d (in der Aussprache [(ə)t]) wird bei ihm explizit festgelegt (S. 28):

En grünräigel foon e schriwwise säit, dåt önj e nütid (praes.) jü iinjing -(e)t schraawen wårt än önj e jütid (praet.) än bait madewurd jütid (perf. part.) - $(e) d$. (Eine Grundregel der Orthographie besagt, dass im Präsens die Endung - $(e) t$ geschrieben wird und im Präteritum und beim Partizip Perfekt - $(e) d$.)

Jörgensen (1972) war die zweite Auflage der Grammatik, die danach noch ein paarmal wiedergedruckt wurde. Die erste Auflage aus dem Jahre 1968 weicht in der Beschreibung des schwachen Verbs in einem entscheidenden Punkt von der späteren Auflage ab. Es heißt hier (Jörgensen 1968, 25):

Bai hu uurde, forålem wan's aw -ne, -re än -we iinje, fålt dat $e$ uk wäch. (Bei einigen Wörtern, vor allem wenn sie auf -ne, -re und -we enden, fällt das $e$ auch weg.)

Was Jörgensen hier also als eine Tendenz bei einigen Verben, vor allem auf -ne, -re und -we, beschreibt, wurde in der zweiten Auflage $\mathrm{zu}$ einer allgemeineren Regel verschärft. Es stellt sich die Frage, wie diese Änderung in Hinblick auf den aktuellen Gebrauch zu interpretieren ist.

Walker (1990, 19-20) folgt in seiner Beschreibung der Konjugation der schwachen Verben Jörgensen (1972). Andresen \& Petersen (1997) ist eine Bearbeitung von Jörgensens Grammatik und die Darstellung der Beugung der regelmäßigen (schwachen) Verben (S. 19-20) basiert im Großen und Ganzen auf dessen Beschreibung. Die VerfasserInnen unterscheiden Verben mit $e$ und ohne $e$ in der 2. und 3. Person Singular Präsens, im Präteritum und im Partizip Perfekt (booge 'wohnen' vs. hiire 'hören') und schreiben: "Wie hiire werden viele Zeitwörter auf -le, -me, -ne, -re, -we sowie alle Zeitwörter auf Selbstlaut $+e$, wie z. B. schraie (weinen) oder büïe (arbeiten) gebeugt." Mit der vagen Formulierung 'viele Verben' kehren die AutorInnen teilweise zu Jörgensen (1968) zurück; welche Verben auf -le, -me, -ne, -re, -we dann nicht wie hiire konjugiert werden, wird offengelassen. 


\section{US WURK LXX (2021), p. 63}

Wilts (1995) gibt in seiner Formenlehre des Bökingharder Friesisch exemplarische Paradigmen von frååge 'fragen' (frååged), hoonle 'handeln' (hooneld) und brüke 'gebrauchen' (brükd), verzichtet aber auf Regeln für die Distribution der Endungen mit und ohne $e$. Dieselben Verben werden in Walker \& Wilts (2001) benutzt, um die Konjugation des Bökingharder Friesisch zu erläutern, auch hier ohne klare Distributionsregeln.

Die Hinweise für die Konjugation der schwachen Verben, die in den Lehrbüchern und Sprachkursen des Mooring gegeben werden, gehen ebenfalls auf Jörgensen (1972) zurück. Auffällig ist aber, dass in Sjölin (1986) und in Petersen (2007) / Petersen \& Laabs (2008) die problematische Bestimmung, dass $e$ bei Verben mit einem Stamm auf $-w$ wegfällt, nicht mehr auftaucht.

Im 'Mooring Uurdebök' (Sjölin et al. 1988) wird zwischen regelmäßigen Verben (r. V.) und unregelmäßigen Verben (unr. V.) unterschieden, ohne dass über diese Unterscheidung in den 'Hinweise[n] für den Benutzer' Rechenschaft abgelegt wird. Verben, die nach der neuen phonologischen Regel flektiert werden, werden aber offensichtlich als regelmäßig bezeichnet. ${ }^{17}$ Als unregelmäßig gelten scheinbar nicht nur starke Verben und schwache Verben mit Stammallomorphie, sondern auch einige Verben mit einem Stamm auf Obstruent, die eine $e$-lose Endung erhalten, wie täiwe und liiwe. Andere Verben aus dieser Gruppe werden aber als regelmäßig eingestuft, nicht nur solche, bei denen es Variation in der Endung gibt (schake 'schicken' - schak $(e) t$ - schak $(e) d$ - schak $(e) d)$, sondern auch solche, die nur ohne $e$ in der Endung vorkommen (brüke 'gebrauchen' brükt-brükd-brükd).

Der Wechsel in der grammatischen Beschreibung des schwachen Verbs, den man beim Vergleich von Bende Bendsen und Tams Jörgensen beobachten kann, weckt die Frage, ob es konkrete Änderungen in der schwachen Verbkonjugation gegeben hat, die dem zu Grunde liegen könnten, ob sich diese Änderungen in der mooringerfriesischen Textüberlieferung nachvollziehen lassen, und ob es diese Änderungen sind, die die Unsicherheit in den neueren Grammatiken ausgelöst haben. Bevor wir versuchen, diese Frage zu beantworten, jedoch vorab einige Bemerkungen zu der Tilgung von Schwa

17. Da Jörgensen (1972) keinen Wegfall von $e$ nach $n g$ vorsieht, könnte das Verb rainge (raingt - raingd - raingd) als unregelmäßig bezeichnet worden sein. Aber rainge könnte auch einfach eine Verschreibung für ränge sein (vgl. Wilts 1995: ränge raingt - raingd - raingd [Ostermooring] neben ringe - rängt - rängd - rängd [Westermooring]); die Verben fänge und önjfänge werden als regelmäßig gekennzeichnet. 


\section{US WURK LXX (2021), p. 64}

nach Sonoranten, die bei der Entwicklung der schwachen Verbkonjugation anscheinend eine wichtige Rolle gespielt hat.

\section{Die Tilgung von Schwa nach Sonoranten}

Der Wegfall von Schwa nach Sonoranten bei Verben der 2. schwachen Klasse scheint ein durchaus üblicher phonologischer Vorgang zu sein; man kann ihn nicht nur im Mooring, sondern auch in den anderen friesischen Mundarten beobachten.

Im Saterfriesischen fällt das Schwa nach den alveolaren Sonoranten $(l, n$, $r$ ) bei Verben der 2. schwachen Klasse weg (Fort 1980, 221, Kramer 1982, 32):

(6)

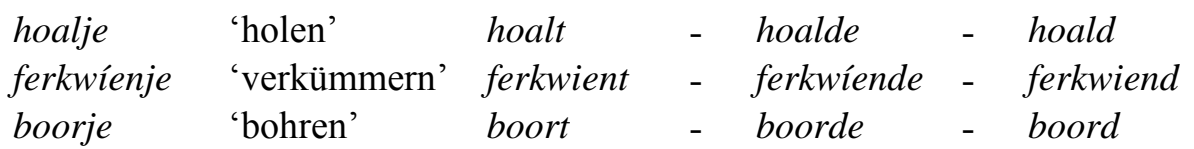

Die 2. schwache Klasse hebt sich aber durch das thematische Element -jeim Infinitiv, in der 1. Person Singular Präsens und im Plural Präsens noch immer deutlich von der 1. schwachen Klasse ab.

Im Wangeroogischen, wo die zwei Konjugationsklassen verschwunden sind und die Distribution der Endungen sich am Stammauslaut orientiert, erscheint die Endung ohne $e$ nach Vokalen, Sonoranten und $z$ (vgl. (7)), die Endung mit $e$ nach den übrigen Konsonanten (Hoekstra 1998, 26-30, Bosse 2012).

$\begin{array}{lll}\text { (7) dau 'tauen' } & - & \text { daut 'taut' } \\ \text { muur 'mauern' } & - & \text { muurd 'gemauert' } \\ \text { rul 'rollen' } & - & \text { ruld 'gerollt' } \\ \text { loo }_{e} \text { 'lohnen' } & - & \text { loo }_{e} \text { ' 'lohnt' } \\ \text { biischoo } m \text { 'beschämen' }_{\text {boo }_{e} z \text { 'irre reden' }} & - & \text { beschoo }_{e} m d \text { 'beschämt' } \\ \text { boo }_{e} \text { ' } & - & \text { boo }_{e} \text { 'redet irre' }\end{array}$

Im Helgoländischen, das noch zwischen Verben der 1. und 2. schwachen Klasse unterscheidet, fällt das $e$ bei Verben der 2. Klasse nach den alveolaren Sonoranten und teilweise nach $s$ weg (Borchert \& Århammar 1987, 99, Århammar 2001, 756):

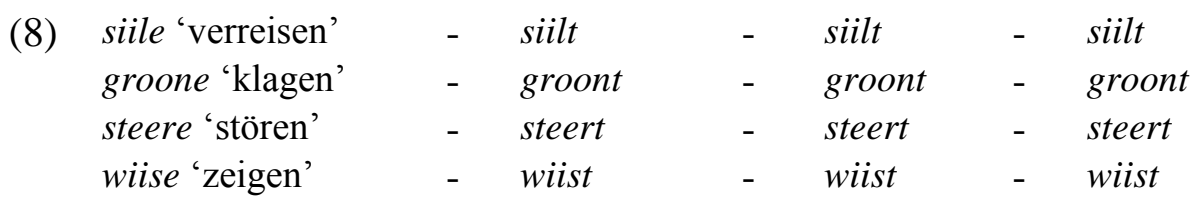




\section{US WURK LXX (2021), p. 65}

Gewährt bleibt der Unterschied zwischen der 1. und 2. schwachen Klasse hier durch den Themavokal - $e$ - im Infinitiv, in der 1. Person Singular Präsens und im Präsens Plural der 2. Klasse, der in der 1. Klasse fehlt.

Und auch in denjenigen festlandnordfriesischen Mundarten, die die Zweiteilung der schwachen Konjugationsklassen beibehalten hatten, dem Mittel- und Südergoesharder Friesischen, war das Schwa der Endung nach Sonoranten weggefallen.

$$
\begin{array}{lllllll}
\text { mgo. hååli 'holen' } & - & \text { håålt } & - & \text { hååld } & - & \text { hååld } \\
\text { sgo. haali 'holen' } & - & \text { haalt } & - & \text { haald } & - & \text { haald }
\end{array}
$$

Auch hier führte dies nicht zu einem Zusammenfall der 1. und 2. schwachen Klasse, da der kennzeichnende Themavokal - $i$ - im Infinitiv, im Präsens Plural und teilweise in der 1. Person Singular Präsens noch vorhanden war.

Diese gleichgerichteten Entwicklungen in den friesischen Mundarten wecken die Frage, wodurch sie motiviert sind: Weshalb nehmen Verben auf Sonorant gerne eine Endung ohne $e$ und Verben auf Obstruent eine Endung mit $e$ ? Dazu muss zunächst bedacht werden, dass die Konsonanten der Verbendungen alveolare Plosive $(-t,-d)$ oder dentale Frikative $(-s t)$ sind. Die alveolaren Sonoranten des Stammes $(l, n, r)$ und die alveolaren (und dentalen) Konsonanten der Endung könnten sich zunächst angezogen haben, was zum Wegfall des zwischenliegenden Schwa führte (vgl. das Saterfriesische). ${ }^{18}$ Anschließend kann die Tilgung von Schwa auf die nicht-alveolaren Sonoranten $(m, n g)$ ausgedehnt worden sein. Der Wegfall von $e$ führte bei Verben auf Sonorant zu einem idealen Ablautcluster (Sonorant + Plosiv/ Frikativ), d.h. einem Cluster mit deutlich sinkender Sonorität. Bei Verben auf Obstruent kann der Wegfall von $e$ vermieden worden sein, weil im sich daraus ergebenden Ablautcluster Plosiv/Frikativ + Plosiv/Frikativ aufeinander stoßen würden, d.h. Konsonanten mit (fast) gleicher Sonorität.

Man kann historisch auch die entgegengesetzte Entwicklung beobachten: eine Tendenz, das Zusammenstoßen von den Alveolaren/Dentalen in Verbstamm und Verbendung gerade $\mathrm{zu}$ vermeiden, möglicherweise um eine größere morphologische Transparenz zu gewährleisten. Eine solche Tendenz könnte das Einfügen eines bedeutungslosen Fugenelements -ig- (moor.

18. Eine ähnliche 'Dentalattraktion' kann man vielleicht bei der westgermanischen Metathese beobachten, bei der $r$ scheinbar von Alveolaren/Dentalen angezogen wurde. Vergleiche moor. gjars Gras', afr. gers <* gras-, moor. foosch 'Frosch' < *foorsch < *frosk-, moor. bjarne 'tränken' < afr. *berna < *brunnjan, amr. beerd 'Fußboden' < afr. *berd <*bred- (hd. Brett), sylt., helg., gurt, schm. go(r)t 'groß' <* grot- < afr. grāt (-[a:]-). 


\section{US WURK LXX (2021), p. 66}

$-i$-) in Verben auf Alveolar/Dental erklären, das man in den festlandestgermanischen Sprachen feststellen kann (Hoekstra 1993). Vergleiche folgende mooringerfriesische Beispiele:

(10) bedårie 'sich beruhigen; zu sich kommen' (vgl. wfr. bedarje ohne -ig-)

kulie 'frieren, Kälte empfinden' (fa. kolige)

schuunie 'schonen'

reedie 'retten'

bääsie 'beten' (fa. beed(i)ge)

lååsie '(ein)laden’ (<*lathigia, afr. lathia)

Nach diesem kurzen Intermezzo über die Tilgung von Schwa nach Sonoranten wenden wir uns jetzt den mooringerfriesischen Texten zu.

\section{Die Entwicklung der schwachen Verbkonjugation in Texten}

In diesem Abschnitt untersuchen wir die Entwicklung der schwachen Verbkonjugation in der mooringerfriesischen Textüberlieferung, die wir dazu in vier Perioden eingeteilt haben: (i) die erste Hälfte des 19. Jahrhunderts (überwiegend das Werk von Bende Bendsen), (ii) die zweite Hälfte des 19. Jahrhundert (bis zum Ende des Ersten Weltkriegs), (iii) die Zeit zwischen dem Ersten und dem Zweiten Weltkrieg und (iv) die Nachkriegszeit bis heute. Materialgrundlage unserer Untersuchung bilden hauptsächlich die digital verfügbaren mooringerfriesischen Texte im Thesaurus des Nordfriesischen, einer Datenbank der Nordfriesischen Wörterbuchstelle der Christian-Albrechts-Universität zu Kiel. ${ }^{19}$

\subsection{Erste Hälfte des 19. Jahrhunderts}

Die grammatische Darstellung der schwachen Verben bei Bende Bendsen (1860) hat schon klar gezeigt, dass bei ihm der Unterschied zwischen der 1. und der 2. schwachen Konjugationsklasse noch vorhanden ist. Eine ausführlichere Untersuchung der schwachen Verbformen in Bendsens grammatischen und literarischen Texten bestätigt, dass er dieses System in seinem Werk sicher anwendet. Es lassen sich Oppositionen zwischen der 1. und der 2. schwachen Klasse nicht nur bei Verben mit stammauslautenden Obstruenten (vgl. (11)), sondern auch bei solchen mit stammauslautenden Sonoranten (vgl. (12)) nachweisen:

19. https://www.frisistik-thesaurus.uni-kiel.de/de 


\section{US WURK LXX (2021), p. 67}

$$
\begin{array}{ll} 
& \text { 1. schwache Klasse } \\
\mathrm{p} & \text { kláppt 'geschnitten' [BB 433] } \\
& \text { stiept 'gezogen (Kerzen)' [BB } \\
& 313] \\
\mathrm{b} & - \\
\mathrm{f} & -{ }^{20} \\
\mathrm{w} & \text { täiwd 'gewartet' [BB 401] } \\
& \text { liewd 'glaubte' [Fortêlling 6] } \\
\mathrm{t} & \text { sêt 'setzte; gesetzt' [BB 501] } \\
\mathrm{d} & - \text { 22 } \\
\mathrm{s} & - \\
\mathrm{z} & \text { räisd 'reiste' [BB 351] } \\
& \text { forlieshd 'entbunden' [BB 417] } \\
& \text { riehst 'erhebt sich' [BB 422] } \\
\mathrm{k} & \text { brückt 'gebraucht' [BB 400] } \\
& \text { röckt 'raucht' [BB 259] } \\
\text { ch } & - \\
\mathrm{g} & \text { bággd 'gebaut' [BB 272] }
\end{array}
$$

$\begin{array}{ll} & \text { 1. schwache Klasse } \\ 1 & \text { têld 'zählte' [BB 297] } \\ \mathrm{r} & \text { hierd 'gehört' [BB 362] } \\ \mathrm{m} & \text { driemd 'träumte' [BB 361] } \\ \mathrm{n} & \text { kānnd 'kannte' [BB 294] } \\ \mathrm{ng} & \text { L }^{24}\end{array}$

2. schwache Klasse

kloppet 'geklopft' [BB 429]

rābbet 'rupft' [BB 260]

${ }^{21}$

làwet 'gelebt' [BB 267]

kōstet 'kostet' [BB 369]

hardet 'härtet' [BB 239]

tühsset 'gezaust' [BB 400]

rōset 'faulte; gefault' [BB 295]

laucket 'schaut' [BB 190]

$-^{23}$

kōget 'gekocht' [BB 261]

2. schwache Klasse spàlet 'gespielt' [BB 263]

wāret 'währte' [BB 427]

sömet 'ziemt' [BB 262]

tienet 'dient' [BB 444]

fānget 'gefangen' [BB 268]

Das Verb fånge 'fangen', das in den inselnordfriesischen Mundarten zur 1. Klasse gehört (fa. fang - fangd), scheint im Mooring zur 2. Klasse gehört zu haben. Dies gilt auch für das zusammengesetzte Verb önjfånge 'anfangen' (BB 399: ás ick üttging, fānget't äujn tó rênen, als ich ausging fing es an zu regnen). Das Mooring schließt sich in dieser Hinsicht den anderen festland-

20. Nur im Stammallomorph $k \bar{a} f$ - des unregelmäßigen Verbs kupen 'kaufen' $-k \bar{a} f t-k \bar{a} f t$ $-k \bar{a} f t$.

21. Zu puffen 'puffen; knallen' (BB 77, 153) gehörte möglicherweise eine flektierte Form * puffet, die aber bei Bendsen nicht überliefert ist (vgl. fa. pofe - pofet).

22. Nur unregelmäßige Verben wie bläjdden 'bluten' - blêt - blêt - blêt und sprijdden 'spreiten' - sprāt-sprāt-sprāt.

23. Zu háchen 'hauchen' (BB 324) gehörte ohne Zweifel eine flektierte Form *háchet, die aber bei Bendsen nicht überliefert ist.

24. Nur unregelmäßige Verben wie lingen 'reichen' - lāngt-lāngd-lāngd. 


\section{US WURK LXX (2021), p. 68}

nordfriesischen Mundarten an, vgl. sgo. (öön)fangi, mgo. (oon)fangi und ngo. (Boy Jacobsen) (aun)fangiä.

Entlehnte Verben mit der Stammerweiterung -iir gehören bei Bendsen der 2. schwachen Klasse an. Vergleiche studiret (BB 421) und regieret (BB 270, 353, 273).

Die Schwa-Epenthese bei Verben mit einem Stamm auf Konsonant + Sonorant vollzieht sich bei Bendsen im Großen und Ganzen wie im heutigen Mooring. Nur bei Verben mit einem Stamm auf -rm gibt es einzelne Abweichungen. Laut Jörgensen (1972: 28) bleibt bei solchen Verben die Schwa-Epenthese aus: wiirme - wiirmt - wiirmd - wiirmd. ${ }^{25}$ Bei Bendsen findet man jedoch drei Beispiele mit einem epenthetischen $e$ : he shwieremt ámbài 'er streift herum', jö stieremt 'sie riecht' ${ }^{26}$ und he hêremt hám 'er härmt sich' (BB 273). Eine Erklärung für den Unterschied zwischen Bendsen und Jörgensen könnte sein, dass das $r$ bei Bendsen noch nicht vokalisiert war, sondern als Zungenspitzen- $r$ realisiert wurde, so dass das auslautende Konsonantencluster $\mathrm{rmt} / \mathrm{rmd}$ damals schwerer auszusprechen war als heute. Auffällig scheint dann, dass das Verb staurmen 'stürmen' durchgehend ohne $e$ zwischen $r$ und $m$ auftritt (BB 214, 263: dāt staurmt). Obwohl Bendsen dies gewöhnlich nicht orthographisch ausdrückt, wurde das epenthetische $e$ hier aber wahrscheinlich zwischen $a u$ und $r$ eingefügt, wie es später z. B. auch noch bei Nis Albrecht Johannsen zu beobachten ist: et lojtet en tunnert en stauermt ma Maacht 'es blitzt und donnert und stürmt mit Macht' (Nis Randers). Dass Bendsen zwischen tautosyllabischem au und $r$ tatsächlich Schwa-Epenthese hatte, wird vielleicht durch kláwwern 'klettern' (BB 252), ick kláwwer 'ich klettere' vs. we kláwre 'wir klettern' (BB 266) bestätigt. ${ }^{27}$

Ein Zeitgenosse und Bekannter Bendsens, der Niebüller Carsten Johannsen, schrieb in den Jahren 1823, 1824 und 1825 drei Königsgedichte (Wilts 1977), in denen u.a. die Verbformen ihret 'ehrt' (afr. äria 'ehren') und fihret 'feiert' (afr. firia 'feiern') mit einem $e$ in der Endung auftreten, im heutigen Mooring iirt und fiirt. ${ }^{28}$

25. Mit analogischem Ausgleich für älteres wiirme - wårmt-wårmd-wårmd.

26. Einmal schreibt Bendsen aber auch stiermd und zwar in seiner Übersetzung des Grimm-Märchens Am de Magandelbuhmm.

27. Das heutige mooringerfriesische klauere 'klettern' hat ein festes $e$ im Stamm.

28. Die Form firret 'feiert' tritt auch schon im ältesten mooringerfriesischen Sprachdokument, dem Königsgedicht des Niebüller Studenten Andreis Volquard aus dem Jahre 1746, auf (Holthausen 1938). 


\section{US WURK LXX (2021), p. 69}

\subsection{Zweite Hälfte des 19. Jahrhunderts}

Die Zweiteilung der schwachen Verben in eine 1. und eine 2. Konjugationsklasse, wie sie sich in Bendsens Werk zeigt, setzt sich im weiteren 19. Jahrhundert fort. In Texten, die etwa zwischen 1850 und 1918 entstanden sind, findet man bei schwachen Verben der 2. Klasse mit einem Stamm auf Sonorant noch immer ein $e$ in der Endung (-est, -et). So in der anonymen 'Frast-Pretjei' (höölet 'heult', haalet 'holt'), ${ }^{29}$ im Märchen 'Da tra Brauderne' von Detlef Nicolai Christiansen (fertienet 'verdient'), sowie in den Gedichten von Johann Jannsen (tinget 'dingt, feilscht'), Friedrich August Feddersen (smelet 'lächelt', wölet 'wühlt'), Beate Godbersen (luunet 'lohnt'), Sönke Petersen (möret 'gemauert', ihret 'ehrt', hantiret 'hantiert', paret 'paart', swaret 'antwortet (entspricht)', ilet 'eilt', halet 'geholt', malet 'malt', römet 'rühmt', ferinet 'vereint', tinet 'dient', lunet 'lohnt') und Hans Andreas Carstensen (forspâlet 'verspielt', grênet 'stöhnt', stênet 'stöhnt', inmöret 'eingemauert', lörret 'wartet', apspirred 'aufkeimt', trāllet 'dreht', tōmmet 'gezähmt'). Bei Carstensen tauchen jedoch auch schon ein paar Beispiele auf, in denen das $e$ nach Sonoranten weggefallen ist (taksiird 'taxiert', tuhuupesnåård 'zusammengeschnürt', shaamd 'schämte', ferspâlt 'verspielt', ferwâlt 'verwelkt', fångd...önj 'fing....an').

Bei (önj)fånge scheint sich der Wegfall von $e$ verhältnismäßig früh durchgesetzt zu haben. Dies geht auch aus den in den Jahren 1879/1880 und 1887 erhobenen Wenkersätzen hervor; in Wenkersatz 3 (...daß die Milch bald an zu kochen fängt) verzeichnet nur der Bogen aus dem Marienkoog für die Bökingharde noch äun...faanget; Deezbüll, Niebüll, Risum und Nordlindholm haben äujnfangt, öinjfangt, an...fangt und önjfangt (Bosse 2019, Kap. 4).

Interessant sind die Belegstellen von Verben der 2. schwachen Klasse mit einem Stamm auf Sonorant in den Sprichwörtern in Moritz Momme Nissens 'Frèske Findling' (1873-1883), die im Mooring (bis auf eine Ausnahme) alle ein $e$ haben:

(13) betalet 'bezahlt' (382, 387, 992, 1109), (ouf)haled 'abholt' (70, 567, 835), spâlet 'spielt' (366, 645, 909), ferspâlet 'verspielt' (532) auersparet 'erspart' (743), waret 'währt' $(701,1162)$, lörret $(835,1057)$ bonet 'flucht' (765), tinet 'dient' (714, 852), fertinet 'verdient' (743) skômet 'geschämt' (47), skômet 'schämt' $(47,778)$

29. In der 'Frast-Pretjei' erscheint auch zum ersten Mal bagget 'baute' (für bagd), eine Form, die sich im jüngeren Mooring stark durchsetzen wird (s. unten). 


\title{
US WURK LXX (2021), p. 70
}

\author{
Vergleiche Sprichwort 743:
}

(14) Erspartes geld ist eben so gut als verdientes. Auerspard gil es èwen sü gödd, as fertint. (Karrh.) Auersparet gil as èwen sü gödd as fertinet. (Moor.) Arspaaret gil es alli sü guid es fortinet. (Wied.) Auerspâret gil es ewen sa göd es fertinet. (Hattst.) Auerspart gil es eben sa god as fertint. (Brekl.) Auersparet gil es ewen sü goud als fertinet (Ockh.)

Das Karrharder und Mittelgoesharder (Breklumer) Friesisch zeigen hier schon den Wegfall von $e$ nach Sonoranten. Im Mittelgoesharder Friesisch wird das $e$ systematisch getilgt, im Karrharder Friesisch bei einigen Verben (auerspard, halt, (fer)spelt, (fer)tint, wart). Im Mooring taucht nur einmal (in Sprichwort 1130) eine Form hald 'holte' ohne $e$ in der Endung auf.

\subsection{Zwischenkriegszeit}

Auch in der Zwischenkriegszeit bleibt das System der zwei schwachen Konjugationsklassen im Wesentlichen unangetastet. In der Geschichte 'Üt baj e Wejle' von Nis Albrecht Johannsen (*1855 in Klockries) finden sich folgende Belege von Verben der 2. Klasse mit einem Stamm auf Sonorant, die ein $e$ in der Endung aufweisen:

(15) ütwölet 'ausgewühlt', weelet 'weilte', betalet 'bezahlt', halet 'holte', spalet 'spielte', smeelet 'lächelte', feelet 'feilte' ${ }^{30}$

waaret 'währte', swaret 'antwortete', regijret 'regiert', stjörret 'steuerte', ${ }^{31}$ lörret 'wartete', verwaaret 'verwahrt', slijret 'streichelte', boret 'bohrte', stijret 'starrte', stijret 'gestört', tohupesnaret 'zusammenschnürte', apklaret 'aufklärt', verijret 'verehrt', sparet 'gespart', verstijret 'verstört', spirret 'keimte'

versömet 'versäumt', stammet ...baj 'stimmte...bei'

vertijnet 'verdient', tijnet 'gedient'

önjfanget 'anfing; angefangen'

Nis Albrecht Johannsen ist der Letzte, der noch durchgängig önjfånget schreibt, andere SchriftstellerInnen in der Zwischenkriegszeit verwenden nur noch önjfångt. Er ist auch der Letzte, der in seinem Werk bei entlehnten

30. Feele 'feilen', ein Verb der 2. schwachen Klasse, steht bei Nis Albrecht Johannsen noch in Opposition zu feele 'füllen', einem Verb der 1. schwachen Klasse.

31. Schon bei Bendsen (Fortêlling 7: stjürret 'lenkte') war stjörre ein Verb der 2. schwachen Klasse, wie saterfr. stjúurje, aber im Gegensatz zu fa. s(t)jüür und wfr. stjoere, die zum 1. schwachen Klasse gehören. 


\section{US WURK LXX (2021), p. 71}

Verben mit der Stammerweiterung -iir (bei ihm ijr-) eine Endung mit $e$ hat (studijret 'studierte', regijret 'regiert' usw.).

Ansonsten ist auch bei anderen AutorInnen in der Zwischenkriegszeit das $e$ der Endung bei schwachen Verben der 2. Klasse noch allgemein vorhanden. In den fünf Theaterstücken von Emil Hansen (*1875 in Lindholm), die wir untersucht haben, fanden wir folgende Belegstellen: ${ }^{32}$

(16) gnurret 'knurrt', slorrest 'schlürfst', slieret 'gestreichelt', lörret 'wartet' spalet 'spielt', feelet 'fehlt', haalest 'holst', haalet 'holt', betaalet 'bezahlt', smeelet 'lächelt'

schomest 'schämst', schomet 'schämt; geschämt', ohmet 'atmet', brummet 'brummt', oufrumet 'abgesahnt', römet ...ap 'räumt...auf'

tienet 'gedient', fertienet 'verdient', stänet 'stöhnt', ohnet 'geahnt'

Nur bei Verben mit der Stammerweiterung -iir wird das $e$ weggelassen (spikeliert 'grübelt', passiert 'passiert', quittiert 'quittiert', studiert 'studiert', triwweliert 'geärgert', disketiert 'diskutierte').

Auch von Katharina Ingwersen (*1879 in Deezbüll) haben wir uns fünf Theaterstücke vorgenommen. ${ }^{33}$ Verben der 2. schwachen Klasse auf Sonorant bekommen in diesen Texten die Endung -et:

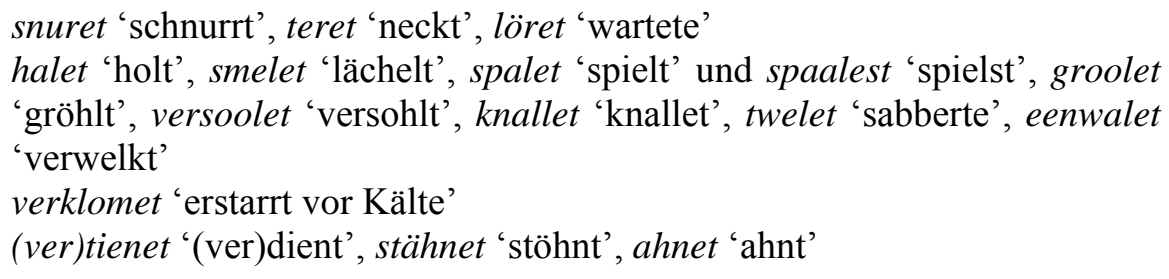

Man findet bei ihr aber nicht nur studiert 'studiert', sondern auch immer sliert 'streichelt', slierd 'streichelte; gestreichelt' (vgl. Johannsen slijret, Hansen slieret).

Im Werk von Albrecht Johannsen (*1888 in Deezbüll), dem Sohn von Nis Albrecht Johannsen, tritt neben den üblichen Formen mit $e$ in der Endung eine wachsende Zahl von Formen ohne $e$ auf, z. B. swaard 'antwortete', smeld 'lächtelte', struld 'strömte', vertiend 'verdient', schomd 'schämte' und studierd 'studiert'.

32. Es handelt sich um die folgenden Theaterstücke: Fidder oder San, Di naje Dokter oder Jü Haingstekur, Browejerw, En bröcket Dünjs, Fogets Feen (alle o.J.).

33. Es handelt sich um die folgenden Theaterstücke: Aaple aw e Hilebuum (o.J.), Jö wall freie än schall aj än jö schall freie än wall aj (1927), Kü heet ferjin, dåt's Kuulew wään (1934), Momme schall er hitje (1949), Et fertiinet lü̈̈nj (1953). 


\section{US WURK LXX (2021), p. 72}

Ein besonderer Fall ist Herrlich Jannsen (*1906 in Neukirchen), die das Mooringer Frasch erst mit 20 Jahren von Katharina Ingwersen lernte und sowohl vor als auch nach dem Zweiten Weltkrieg publizierte. Auch bei ihr ist das alte System der beiden Konjugationsklassen im Prinzip erhalten, aber es macht sich der Wegfall von Schwa nach Sonoranten schon bemerkbar. In ihrer Autobiographie 'Häl en junk' (die von 1956 bis 1959 im Südtondern Tageblatt veröffentlicht wurde) findet man z. B. neben löret 'wartete', teret 'neckt; neckte; geneckt', lunet 'lohnt; gelohnt', tiinet 'dient; gedient', fertiinet 'verdient', stäänet 'stöhnte', fermoonet 'vermahnt', schaanet 'buttert', spaalet 'spielt; spielte; gespielt', betaalet 'bezahlt', maalet 'malte; gemalt', smeelet 'lächelt; lächelte' und schomet 'schämte' Formen wie sliirt 'streichelte', regiirt 'regiert', ferklaart 'erklärt', spaart 'gespart', swaart 'antwortete' (1x swaaret) und waart 'währt'. Darüber hinaus manifestiert sich bei ihr jedoch eine starke Neigung zur Übergeneralisierung der $e$-Endung, die wohl auf sprachliche Unsicherheit zurückzuführen ist. Man findet in 'Häl en junk' nicht nur ein $e$ bei Verben der 1. schwachen Klasse auf Obstruent (täiwet 'wartete' statt täiwd, liiwet 'glaubt; geglaubt' statt liiwt) liiwd, schäket 'geschickt' statt schäkd) - also in Kontexten, wo dies, als die phonologische Distribution Oberhand gewinnt, auch durchaus bei anderen $\mathrm{zu}$ beobachten ist (s. Abschnitt 5.4) - sondern ebenfalls bei Verben der 1. schwachen Klasse auf Sonorant (fäilet 'fühlt' statt fäilt, späilet 'spült' statt späilt, apdiilet 'aufgeteilt' statt apdiiljd, ferteelet 'erzählte' statt ferteeld, staalet 'stellt' statt stalt, beganet 'begann' statt begand). ${ }^{34}$

\subsection{Nach dem Zweiten Weltkrieg bis heute}

Nach dem Zweiten Weltkrieg setzt sich der Wegfall von Schwa nach Sonoranten durch. Dies passiert aber schleichend und nicht ohne Ausnahmen. Wir haben schon gesehen, dass ältere AutorInnen wie Herrlich Jannsen in ihren Publikationen nach dem Krieg grundsätzlich noch am alten System festhalten. Auch in den Erzählungen von Elise Heitmann (*1909 in Emmelsbüll), ${ }^{35}$ die wie Jannsen aus der Wiedingharde stammte, findet man bei Verben der 2. schwachen Klasse auf Sonorant noch oft ein $e$ (mååled 'gemalt', smeeled 'lächelte', fertrooled 'verzauberte; verzaubert'; gnured

34. Auch Verben mit einem Stamm auf Vokal erhalten in Herrlich Jannsens Texten oft ein hyperkorrektes $e$ : rouet 'ruht' (statt rout), schaiet 'geschieht' (statt schait), biiet 'biegt, beugt' (statt biit), woorschouet 'warnt' (statt woorschout), bü̈̈jet 'arbeitete; gearbeitet' (statt bü̈id).

35. Es handelt sich um folgende Texte: Di ü̈̈lje Ford, Foont lönj und Butegrapeln. 


\section{US WURK LXX (2021), p. 73}

'knurrte', löred 'wartete; gewartet'), aber auch Variation (hååld 'holte' neben ferhååled 'erholt', wööld 'wühlte' neben wööled, swåård 'antwortete' neben swååred, sliird 'streichelte' neben sliired) und Formen, die nur ohne $e$ vorkommen (bewård 'bewahrt', ferwård 'verwahrt', fermoond 'vermahnt' sowie Verben mit der Stammerweiterung -iir wie studiird 'studierte', operiird 'operiert' und kommandiird 'kommandierte'). ${ }^{36}$

Bei der produktiven Schriftstellerin Marie Tångeberg (*1924 im Marienkoog) hat sich der Wegfall von Schwa nach Sonoranten im Prinzip vollzogen, aber es tauchen in ihrem Werk noch vereinzelte Formen mit $e$ auf. In ihrer Astrid-Lindgren-Übersetzung 'Eemil foon Lönebärj' (Tångeberg $1993^{2}$ ) findet man:

(18) bruuld 'brüllte', swaalden 'Heu machten', spaald(en) 'spielte(n)', smeeld 'lächelte', hååld 'holte'

aber: rü̈̈djmååled 'rotgemalt', fertrooled 'verzaubert'

schörden 'scheuerten', schört 'scheuert', spikeliird(en) 'grübelte(n)', lörd(en) 'warteten', swåård 'antwortete'

aber: wååred 'währte', löred än löred 'wartete und wartete'

fertiinjd 'verdient'

aber: naischarned bööder 'neugebutterte Butter'

Einige wenige Verben der 2. schwachen Klasse auf Sonoranten scheinen tendenziell stärker am $e$ festgehalten zu haben, darunter möglicherweise Verben mit kurzem Stammvokal $+r$ wie löre 'warten', gnure 'knurren', tareltäre 'necken' und einzelne Verben wie boone 'fluchen', oome 'atmen', prunge 'tauschen'. Von den letzten drei Verben haben wir im Material jedenfalls keine Formen ohne $e$ gefunden. Ein klares Muster beim Wegfall oder auch beim Erhalt von $e$ ist aber in den Texten nicht zu erkennen, so dass bei der Verbreitung der Tilgung von $-e$ nach Sonoranten wohl am ehesten an lexikalische Diffusion zu denken ist.

Als das Schwa nach Sonoranten nachgerade verschwand, entfiel damit ein Teil der Grundlage für die Verteilung der schwachen Verben nach Konjugationsklassen, der Themavokal $-e$ - wurde als Teil der Endungen reanalysiert und die Distribution der Endungen -(e)st, -et und - $(e) d$-richtete sich zunehmend nach dem Stammauslaut des Verbs. Es blieben nur die Verben der 1. schwachen Klasse mit einem Stamm auf Obstruent (vgl. (5))

36. Bei Heitmann finden sich auch hyperkorrekte Formen wie späiled 'spülte', pliired 'blinzelte', fååred 'fuhr' und laaned 'lehnte' statt zu erwartenden späild, pliird, fård, laand (späile, pliire, fååre und laane sind ursprünglich Verben der 1. schwachen Klasse). Die Form späiled hatten wir auch schon bei Herrlich Jannsen angetroffen. 


\section{US WURK LXX (2021), p. 74}

als Ausnahmen der phonologischen Distribution der Endungen. Man braucht sich deshalb kaum zu wundern, dass diese kleine Gruppe von Ausnahmen sehr anfällig für analogischen Ausgleich ist. Swiipe 'wickeln', bei Bendsen noch ein Verb ohne $e$ ('En jüttläujnsh Tähl': inswiept äujn en tjokken Māntel 'eingewickelt in einen dicken Mantel'), kommt sonst im Mooring nur mit $e$ vor. Das Verb bage 'bauen', das einzige Verb der 1. Klasse mit einem auslautenden $g$, bekommt heutzutage allgemein die regelmäßigen Endungen (baget - baged - baged). Eine Form bagget ist schon in der 'Frast-Pretjei' (1858) überliefert und Nis Albrecht Johannsen scheint der Letzte zu sein, der in seinen Texten noch baggt/baggd (Ostermooring) bzw. beggt/beggd (Westermooring) ohne $e$ verwendet. Bei anderen Verben konkurrieren die regelmäßigen Endungen mit $e$ mehr oder weniger stark mit althergebrachten Endungen ohne $e$. Einige dieser $e$-Formen haben auch schon eine längere Geschichte. Das Verb mårke 'merken' hatte bereits bei Bendsen ein $e$ (BB 204: He hêt wát mārket, er hat etwas gemerkt) sowie auch bei Nis Albrecht Johannsen (market) und Emil Hansen (maarket). Daneben stand aber auch schon früh eine Form ohne $e, \mathrm{z}$. B. bei Katharina Ingwersen (maarkt). ${ }^{37}$ Nis Albrecht Johannsen schreibt bereits lijset neben lijsd (zu liise 'lösen') und rijset neben rijsd (zu riise 'erheben') und bei Mattgetrehn Jans Hamsen (1929) findet man zum ersten Mal (?) schacket (zu schake 'schicken'). Rejset/räjset (zu räise 'reisen) ist bei Emil Hansen und Katharina Ingwersen belegt; Nis Albrecht Johannsen schreibt noch meistens rejsd/räjsd. Nis Albrecht Johannsen hat bei stjarte 'stürzen' noch die Formen ohne $e$ (stjart - stjart - stjart), aber Formen mit $e$ (stjartet - stjarted - stjarted) erscheinen schon in der Zwischenkriegszeit (z. B. bei seinem Sohn Albrecht Johannsen). ${ }^{38}$ Andere $e$-Formen kommen jedoch anscheinend erst nach dem Zweiten Weltkrieg neben den $e$-losen Formen auf. Vergleiche: ${ }^{39}$

37. Möglicherweise sind hier zwei Verben durcheinandergeraten: mårke im Sinne von 'bemerken' (ursprünglich ein Verb der 1. schwachen Klasse) und mårke im Sinne von 'mit einer Marke versehen' (ursprünglich ein Verb der 2. schwachen Klasse).

38. Schon Bende Bendsen verwendet eine Form mit $e$ in der Volkserzählung 'En jüttläujnsh Tähl': Bast'r ock fullkàmen wáss àw, dat din Kö ai unnerwäis stjartet, ihr dö to Ranners kámst? 'Bist du auch ganz sicher, dass deine Kuh nicht unterwegs stürzt, bevor du zu Randers kommst'.

39. Das jüngere niederdeutsche Lehnwort smööke 'rauchen' ist Ende des 19. Jahrhunderts ein Verb der 1. schwachen Klasse (Nissen [1873-1883], Sprichwort 841: He smökt, as wann er an lattjen mon bagt 'Er raucht, als wenn da ein kleiner Mann backt'), bleibt es in der Zwischenkriegszeit (Ingwersen, Sär ülj Fomene: Hi smökt sö hal 'Er raucht so gerne'), aber erhält nach dem Zweiten Weltkrieg vermehrt ein $e$ in der Endung (vgl. 


\section{US WURK LXX (2021), p. 75}

(19) täiwe 'warten'

Wi heewe ål fiirng ålte lung täiwed (Nommensen 1988, 20)

'Wir haben schon viel zu lange gewartet'

liiwe 'glauben'

Ik liiwed, dåt dåt en sääkern transport wus (Carsten Boysen, Rucht än rucht)

'Ich glaubte, dass es ein sicherer Transport war'

klape 'schneiden'

di trädj as döörklaped wörden (Tångeberg 2020, 72)

'der Faden ist durchgeschnitten worden'

kape 'kippen'

e kaferuume amkaped (Tångeberg $\left.1993^{2}, 10\right)^{40}$

'die Kaffesahne umgekippt'

snåpe 'schnappen'

as ik de snåped (Carsten Boysen, Rucht än rucht)

'als ich dich schnappte'

streeke 'strecken'

Ma ütstreekede ärme (Heitmann 1958, 40)

'mit ausgestreckten Armen'

teeke 'Dach decken'

we schöljn nai teeket heewe (Jannsen, Häl än junk)

'Bei uns sollte das Dach neu gedeckt werden'

fleete 'umziehen' (Jörgensen (1972) und Wilts (1995): fleet - fleetet - fleet

-fleet).

Ouf än tu fleetet uk en ferfläägen påår en gou stak banelönjs än breet deer (Tångeberg 1992, 56)

'Ab und zu zieht auch ein verirrtes Paar ein gehöriges Stück ins Landesinnere und brütet dort'

Hi fleeted e stölj wat näre tut beed (Tångeberg 2020, 77)

'Er rückte den Stuhl etwas näher zum Bett'

mast 'vermissen' (Sjölin et al. (1988): maste - mast - masted - mast)

Thea häi eentlik foole masted önjt laawen (Gonnsen 1993, 69)

'Thea hatte eigentlich viel vermisst im Leben'

Nur ein paar wenige (sehr häufig vorkommende) Verben wie brüke 'gebrauchen' und seete 'setzen' scheinen bisher völlig resistent zu sein; For-

Tångeberg [2020, 85]: liifst smööked hi ouderföljks sigaare 'am liebsten rauchte er die Zigarren anderer Leute').

40. Aber: än sü kapt hi 'und dann kippt er' (S. 42). 


\section{US WURK LXX (2021), p. 76}

men wie *brüke $(s) t / *$ brüked und $*$ seete $(s) t / *$ seeted haben wir in unserem Material nicht gefunden. ${ }^{41}$

Sogar die 2. und 3. Person Singular Präsens-Formen der starken Verben können eine Anpassung an die phonologische Verteilung zeigen, insbesondere wenn sie denselben Stamm wie der Infinitiv haben. Vergleiche:

bat 'bittet' (BB 302: bádt) - badet (Jörgensen 1972, Wilts 1995, Andresen $\&$ Petersen 1997)

jü ... badet ham, mör tu ferteelen (Gonnsen 1993, 41)

'sie bittet ihn, mehr zu erzählen'

tjarscht 'drischt' (BB 303) - tjarschet (Ingwersen \& Johannsen 1928, 14, Jörgensen 1972, Wilts 1995)

wåkst 'wächst' (Ingwersen \& Johannsen 1928, 14: wokst) - wåkset

(Jörgensen 1972, Wilts 1995, Andresen \& Petersen 1997)

deer wåkset wiitje (Gonnsen 1993, 58)

'dort wächst Weizen'

schrumpt 'schrumpft' (BB 304, Andresen \& Petersen 1997) - schrumpet

(Jörgensen 1972, Andresen \& Petersen 1997)

smult 'schmilzt' (BB 304) ${ }^{42}$ - smultet (Jörgensen 1972, Wilts 1995,

Andresen \& Petersen 1997)

leeft 'hebt' (BB 304, Jörgensen 1972, Wilts 1995, Andresen \& Petersen

1997) - leeftet

Lene leeftet harn äärm (Gonnsen, Lene, Bäne än Hansiine)

'Lene hebt ihren Arm'

dreecht 'trägt' (BB 303, Jörgensen 1972, Wilts 1995, Andresen \& Petersen

1997) - dreeget

Di, wat da broudernes lååste dreeget (Heitmann 1973, 73)

'Der, der die Laste der Brüder trägt'

leest 'liest' (BB 303, Jörgensen 1972, Wilts 1995, Andresen \& Petersen

1997) - leeset

Wat leeset hi, man hiire? (Nommensen, 1985, 54)

41. Verben auf - $t$ wie seete 'setzen', fleete 'umziehen' und kneete 'knoten' werden gewöhnlich in die Liste der unregelmäßigen Verben aufgenommen (Jörgensen 1972, Wilts 1995, Andresen \& Petersen 1997). Verben wie swate 'schwitzen' (swat - swat swat), stjarte 'stürzen' und maste 'missen', die im Prinzip nach demselben Muster konjugiert werden, erscheinen dort aber meistens ebenso wenig wie die anderen Verben auf Obstruent mit einer $e$-losen Endung (nur bei Andresen \& Petsersen [1997] ist stjarte - stjart - stjart - stjart in der Liste der unregelmäßigen Verben gelandet).

42. So auch noch in Hans Andreas Carstensens Schiller-Übersetzung 'Dāt līt fōn-e klōk' (1891): sü smult-et $\bar{a} l$ 'so schmilzt es schon'. Bende Bendsen benutzt aber in den Beispielen die Form smoltet: De Snie smoltet,... he smoltet Blie 'Der Schnee schmilzt, ... er schmilzt Blei' (BB 261) 


\title{
US WURK LXX (2021), p. 77
}

\author{
'was lesen Sie (liest er), mein Herr?' \\ greeft 'gräbt' (BB 303, Jörgensen 1972, Wilts 1995, Andresen \& Petersen \\ 1997) - greewet \\ wilt et wååder greewet (Tångeberg 1995, 16) \\ 'während das Wasser gräbt'
}

Die fortschreitende Anpassung an die phonologische Distributionsregel führt zu einer gehörigen Variation und einer starken Unsicherheit bei der Konjugation des (schwachen) Verbs im Mooringer Friesisch. Es ist wahrscheinlich diese Unsicherheit, die sich auch in den Beschreibungen in den Grammatiken und Lehrwerken nach dem Zweiten Weltkrieg widerspiegelt. Zusätzlich kompliziert wird die Lage durch zwei weitere entgegengesetzte Entwicklungen: eine phonetische Tendenz, das $e$ der Endung bei Verben auf Obstruenten in nicht oder weniger betonter Position zu 'verschlucken', und eine Neigung, die 'friesische' Endung mit $e$ hyperkorrekt einzusetzen. Das letzte Phänomen, das wir schon sehr ausgeprägt bei Herrlich Jansen haben beobachten können, wird in einer Situation, in der die Sprache nur noch vereinzelt weitergegeben und oft erst später erlernt wird und in der die Norm einer homogenen Sprachgemeinschaft fehlt, sicherlich immer häufiger vorkommen.

Der Wegfall von $e$ auch nach Obstruenten manifestiert sich zunächst im Präteritum Plural (boogeden 'wohnten' > boog'den) und im flektierten Partizip Perfekt (roosede 'verfaulte' > roos'de), wo es ein klarer Fall rhythmisch oder metrisch bedingter Synkope ist. Die ersten Belege finden sich schon in der Zwischenkriegszeit, z. B. boogten 'wohnten', ki(e)kten 'guckten' (neben ki(e)ket, 1x kiekt) und en bekraansten Wäun 'ein bekranzter Wagen' bei Thusnelda Jannsen (1921) oder auch im Werk von Albrecht Johannsen: bogden 'wohnten', fragden 'fragten', magden 'machten', lakden 'lachten', löckten 'schauten', sackden 'sackten', stopden 'stopften' und gniesden 'grinsden, schnitten Fratzen'. In Texten nach dem Zweiten Weltkrieg tritt dieses Phänomen aber vermehrt auf. Außerdem wird das $e$ jetzt auch in der 2. und 3. Person Singular Präsens, im Präteritum Singular und im Partizip Perfekt (in weniger betonten Satzpositionen?) immer öfter weggelassen. In 'Eemil foon Lönebärj' von Marie Tångeberg findet man: apstoowde buune 'gestowte Bohnen' (aber: apstoowed ääse 'gestowtes Essen'), pååsden 'passten', mölkden 'melkten', schrubden 'schrubbten' (auch: schrubd 'geschrubt'), gjarsden 'grasten', kiikden 'guckten' (neben kiiked(en) 'guckte(n)'), piswisden 'flüsterten' (aber: piswised 'flüsterte'). In den Erzählungen von August Gonnsen (*1921 in Klockries) begegnen z. B. neben snååkden 'redeten' (neben snååked) auch bloosd 


\section{US WURK LXX (2021), p. 78}

'blies', pååsd 'passte', hoowd 'hoffte' (neben hoowed), belaawd 'erlebt' (neben belaawed) und drååwd 'traf; getroffen' (neben drååwed). Solange solche Formen noch neben den vollen Formen vorkommen, könnte man sie als phonetische (möglicherweise betonungsbedingte) Oberflächenrealisierungen der letzteren auffassen und es empfähle sich in diesem Fall, sie mit einem Apostroph zu schreiben (boog'd, boog'den), eine Schreibweise, der man in der Literatur auch ab und zu begegnen kann. Es ist aber klar, dass dieser (optionale?) Wegfall von $e$ nach Obstruenten der letzte Schritt auf dem Weg zu einer Konjugation mit $e$-losen Endungen sein könnte, wie man sie im Prinzip im Niederdeutschen und Hochdeutschen hat, und Einfluss dieser beiden Sprachen ist bei diesem Phänomen auch keineswegs auszuschließen. $^{43}$

Interferenz mit dem Nieder- oder Hochdeutschen liegt auf jeden Fall bei einem zusätzlichen Sprachwandelphänomen im jüngeren Mooring vor, dem Gebrauch der Präteritumendung - $(e) d e$. Im Mooringer Friesisch gibt es bei den schwachen Verben auf Obstruenten (abgesehen von der künstlichen Unterscheidung zwischen $-(e) t$ und $-(e) d$ in der heutigen Orthographie) keinen Unterschied zwischen Formen der 3. Person Singular Präsens und Präteritum (siehe die Beispiele in [1]) und auch bei Verben auf Sonoranten kann der Unterschied durch jüngere Auslautverhärtung verloren gegangen sein. Um das Präteritum eindeutig zu markieren, greifen vereinzelte SprecherInnen deshalb zu einer erweiterten Endung -(e)de ([(ə)tə]. So findet man in den handschriftlichen Tagebuchnotizen von Kurt foon e Borj (= Kurt Jannsen, *1926 in Deezbüll-Burg) durchgängig Formen auf -te wie miinte 'meinte' (für miind), fångte...önj 'fing....an' (für fångd...önj), lörte 'wartete' (für lörd), snååkte 'redete' (für snååked) und boogte 'wohnte' (für booged), fast immer mit Synkope von $e$ (aber neben måågte auch måågete 'machte' für mååged). Nicht nur bei den regelmäßigen, sondern auch bei unregelmäßigen schwachen Verben mit zusammengefallener 3. Person Singular Präsens und Präteritum tritt bei ihm diese Endung auf, vgl. kjarte 'fuhr' (vgl. kääre - kjart-kjard), långte 'reichte' (vgl. linge - långt-långd). Sogar in Fällen, in denen Präsens und Präteritum unterschiedlich sind, wo aber das Deutsche -te hat, wird dies kopiert, vgl. broochte 'brachte' (vgl. bringe

43. Århammar (1995) erwähnt in seinem Vortragstext tatsächlich eine 40-jährige Lindholmerin, die wie im Deutschen ein $e$ in der Endung nach $t$ und $d$ hat, sonst aber nur noch Formen wie laaft 'lebt', biilkt 'ruft', wist 'zeigt' und maut 'macht' verwendet. (Die heute relativ häufig vorkommende Form maue für mååge 'machen' [auch fraue für frååge 'fragen' und jaue für jååge 'jagen'] taucht zum ersten Mal schriftlich bei Mattgetrehn Jans Hamsen (1929) auf.) 


\section{US WURK LXX (2021), p. 79}

- brängt - broocht $).{ }^{44}$ Auf den Wegfall von $e$ nach Obstruenten und den Gebrauch der Präteritumendung - $(e) d e$ kann hier nur am Rande hingewiesen werden, eine systematische Untersuchung dieser Phänomene wäre aber dringend erforderlich.

In den 2017 herausgegebenen Texten von Erk Petersen $*^{*} 1946$ in Niebüll) findet man die heutige Konjugation des regelmäßigen schwachen Verbs im Mooringer Friesisch noch einmal in komprimierter Form. Nach schwachen Verben auf Sonoranten, nicht nur der ehemaligen 1., sondern auch der alten 2. Klasse, findet man eine Endung ohne $e$ (spaald 'spielte', smeeld 'lächelte', hååld 'holte', lörd 'wartete', wåård 'währte', sliird 'streichelte', inspiriird 'inspiriert', tiind 'diente', feriind 'vereint', ståmd 'stammte'), nach Verben der ehemaligen 2. Klasse auf Obstruenten eine Endung mit $e$ (mååged 'machte' usw.). Verben der 1. schwachen Klasse auf Obstruenten halten an ihrer $e$-losen Endung fest (brükd 'gebrauchte', liiwd 'glaubte', schäkd 'schickte'), erscheinen sowohl ohne als auch mit $e$ (mårkd 'merkte' - mårked, liist 'löste' - liised) oder kommen nur mit $e$ vor (täiwed 'wartete'). Das $e$ der Endung bei Verben auf Obstruenten kann wegfallen (mååged 'machte' - måågd, boogeden 'wohnten' - boogden, kiiked(en) 'guckte(n)' - kiikd(en), klooped 'klopfte' - kloopd, snååked 'redete' snååkd, biiljked 'rief' - biiljkd, påked 'packte' - påkt 'packt', pååseden 'passten'-pååsden).

\section{Fazit}

Begonnen haben wir diesen Aufsatz mit einigen kritischen Anmerkungen zur Grammatik von Tams Jörgensen (1972). Am Schluss unserer Untersuchung muss Tams Jörgensen jedoch das Lob gezollt werden, als Erster erkannt zu haben, dass die Konjugation der regelmäßigen schwachen Verben im neueren Mooringer Friesisch auf der Grundlage ihrer lautlichen Beschaffenheit bestimmt wird: Verben mit einem Stamm auf Obstruent erhalten die Endungen - est, -et und -ed, während Verben mit einem Stamm auf Vokal oder Sonorant die $e$-losen Endungen $-s t$, $-t$ und $-d$ bekommen. Durch den Wegfall von Schwa nach Sonoranten, der sich schon im 19. Jahrhundert andeutete und sich dann im Laufe des 20. Jahrhunderts, vor allem nach dem Zweiten Weltkrieg, weiter vollzog, war die Verteilung nach den klassischen zwei Konjugationsklassen, wie diese noch bei Bende Bendsen

44. Århammar (1995) hatte schon auf Präteritumformen wie stiirmde 'roch' (für stiirmd), lökete 'guckte' (für löked) und broochte 'brachte' (für broocht) bei Thea Hermannsen im 'Mooringer Krädjer' (Nr. 7-9) hingewiesen. 


\section{US WURK LXX (2021), p. 80}

und bis weit ins 20. Jahrhundert Gültigkeit hatte, ins Wanken geraten. Oder wie es Århammar $(2001,756)$ unter Verweis auf seinen ungedruckten Vortrag (Århammar 1995) in Bezug auf das gesamte Festlandnordfriesische ausdrückt:

Eine ähnlich konditionierte Synkope [d.h. eine Wegfall von Schwa nach Sonoranten wie im Helgoländischen] erfolgte auch in den Festlandsdialekten, zuerst in den südl. und mittleren. Der alte Unterschied zwischen den Verba der 1. und 2. sw. Konjugation (kein Mittelsilbenvokal bzw. $-e-)$ ist hier im 20. Jh. weitgehend einer rein positionsbedingten Distribution von $-e$ - und $-\varnothing$ - gewichen.

Was Jörgensen (1972) und die anschließende Literatur jedoch übersehen oder allenfalls nicht genügend explizitiert haben, ist die Tatsache, dass noch eine Restgruppe von Ausnahmen zur phonologischen Distributionsregel übergeblieben war. Neben ein paar vereinzelten Verben der ehemaligen 2. schwachen Klasse mit einem Stamm auf Sonorant, die am $e$ festgehalten haben, handelt es sich dabei vor allem um eine Gruppe von Verben der ehemaligen 1. schwachen Klasse mit einem Stamm auf Obstruent, die eine $e$ lose Endung erhält. Dass es sich hier um eine Restgruppe handelt, geht aus der Tatsache hervor, dass sie tendenziell abgebaut wird. Die didaktische Lehre, die man hieraus für die Zukunft ziehen kann, besteht darin, dass diese Gruppe von Ausnahmen in Grammatiken und Lehrbüchern explizit Erwähnung finden und in die Liste der unregelmäßigen Verben mit aufgenommen werden sollte. Zusätzliche Komplikationen bei der Konjugation des schwachen Verbs bilden nicht nur der hyperkorrekte Gebrauch der Endung mit $e$ durch 'neue' SprecherInnen, sondern insbesondere auch der (optionale, betonungsbedingte?) Wegfall von $e$ nach Obstruenten und die Verwendung einer erweiterten Endung - $(e) d e$ in der 3. Person Singular Präteritum. Diese Phänomene verdienen eine nähere Untersuchung, vorzugsweise in einer umfassenden Erhebung zum Gebrauch der schwachen Verbkonjugation in der heutigen gesprochenen Sprache. ${ }^{45}$ In weiteren

45. Nähere Betrachtung verdienen auch die denominativen Adjektive mit dem Suffix $-(e) d$, das zwar eine andere Herkunft hat, aber mit der Partizip-Perfekt-Endung - $(e) d$ identifiziert wurde (vgl. Faltings 1996). Im 'Frasch Uurdebök' (Sjölin et al. 1988) sind Beispiele wie weenuuget 'blauäugig', måålhoodet 'tollköpfig', lunguuret 'langohrig', und saagelbiinet 'sichelbeinig, o-beinig' wahrscheinlich ohne Weiteres mit der Endung -et aus älteren Quellen (BB 171-174) übernommen. Vereinzelte Beispiele wie lungfaingerd 'langfingerig', brünhäärt (-d?) 'braunhaarig' oder fjouerbiind 'vierbeinig' scheinen jedoch nahe zu legen, dass auch hier der Wegfall von Schwa nach Sonoranten gewirkt hat. 


\section{US WURK LXX (2021), p. 81}

Untersuchungen der historischen Entwicklung der schwachen Konjugation müssten schließlich auch die anderen (nördlichen) festlandnordfriesischen Mundarten mit in den Blick genommen werden. ${ }^{46}$

Abkürzungen: afr. = altfriesisch, amr. = amrumerfriesisch, fa. $=$ föhramrumerfriesisch, helg. = helgoländisch, mgo. = mittelgoesharderfriesisch, moor. $=$ mooringerfriesisch, ngo. $=$ nordergoeshardisch, saterfr. $=$ saterfriesisch, sch. = schiermonnikoogisch, sgo. = südergoesharderfriesisch, sylt. $=$ sylterfriesisch, wfr. $=$ westfriesisch

Institut für Skandinavistik, Frisistik und Allgemeine Sprachwissenschaft

- Frisistik -

Christian-Albrechts-Universität zu Kiel

\section{LITERATUR}

Århammar, Nils. 1995. Zwei Beiträge zur nordfriesischen Morphologie: (I) Entstehung und Beseitigung der Homonymie jam 'euch' jam 'ihnen, sie'; (II) Was war und ist "unregelmäßig" in der Flexion der festl-nfr. schwachen Verben? Ungedruckter Vortrag.

Århammar, Nils. 2001. Grundzüge nordfriesischer Sprachgeschichte. In: Horst H. Munske et al. (Hrsg.), Handbuch des Friesischen / Handbook of Frisian Studies. Tübingen: Niemeyer, 744-765.

Andresen, Hayo \& Adeline Petersen. 1997. Kleine Friesische Sprachlehre. Mooringer Mundart. Zusammengestellt von einem Arbeitskreis von Friesischlehrern nach V. Tams Jörgensen, "Kort spräkeliir foon dåt Mooringer frasch". Bearbeitet von Hayo Andresen und Adeline Petersen. Bräist/Bredstedt: Verlag Nordfriisk Instituut.

Bauer, Erika. 1925. Die moringer Mundart. Laut- und Formenlehre nebst Sprachproben. Ein Beitrag zur nordfriesischen Dialektforschung. Heidelberg: Winter.

Bendsen, Bende (BB). 1860. Die nordfriesische Sprache nach der moringer Mundart, zur Vergleichung mit den verwandten Sprachen und Mundarten. Herausgegeben von Dr. M. de Vries. Leiden: Brill.

46. Diese breitere Perspektive hatte Nils Århammar in seinem Vortrag (1995) schon eingenommen. 


\section{US WURK LXX (2021), p. 82}

Borchert, Mina, Nils \& Ritva Århammar. 1987. Wi lear Halunder. Helgoländisches Lehrbuch. Herausgegeben vom Verein zum Wiederaufbau des früheren Helgoländer Nordseemuseums e. V., Helgoland.

Bosse, Temmo. 2012. Wangeroogische $i$-Verben. Betrachtungen zum Verbsystem des ausgestorbenen ostfriesischen Dialekts der Insel Wangerooge. Us Wurk 61, 125-141.

Bosse, Temmo. 2013. Die Entwicklung der schwachen Verben in den nördlichen Mundarten des nordfriesischen Festlandes, ausgehend vom nordergoesharderfriesischen Dialekt. Masterarbeit im Zwei-Fächer-Masterstudiengang, Fach Friesische Philologie der Philosophischen Fakultät der Christian-Albrechts-Universität zu Kiel.

Bosse, Temmo. 2019. Das nord- und ostfriesische Wenkermaterial. Hintergründe, Validität und Erkenntniswert. Doktorarbeit Fach Frisistik, Christian-Albrechts-Universität zu Kiel.

Boysen, Carsten. o. J. Rucht än rucht - ål as huum et namt! (Eefter en tjüsch stok). Hs. Nordfriesische Wörterbuchstelle der Christian-Albrechts-Universität zu Kiel.

Bremmer, Rolf H. 2009. Introduction to Old Frisian. History, Grammar, Reader, Glossary. Amsterdam/Philadelphia: Benjamins.

Dyk, Siebren. 2020. Weak verbs. Taalportaal. https://www.taalportaal.org/taalportaal/topic/pid/topic13998813312987623 (letzter Zugriff am 15.9.2020).

Faltings, Volkert F. 1996. Zur Bildung desubstantivischer Adjektiva mit dem Derivationssuffix -ed/-et im Friesischen und in verwandten Sprachen. Us Wurk 45, 79-113.

Feitsma, Tony. 1990. Udgivelsen af Bendsens Sproglære. Nye fund verdrørende udgivelsen af Outzens glossarium og Bendsens grammatik. Zeittafel und Zusammenfassung auf deutsch. In: Tony Feitsma \& Ommo Wilts (Hrsg.), Bende Bendsen (1787-1875), Grammatiker und Magnetiseur. Mit einem Anhang seiner Märchenübersetzungen. Co-Frisica IX. Kiel: Fach Friesische Philologie/Amsterdam: Stúdzjerjochting Frysk Vrije Universiteit.

Fort, Marron C. 1980. Saterfriesisches Wörterbuch, mit einer grammatischen Übersicht. Hamburg: Buske.

Fortêlling = Peter Jørgensen (Hrsg.). 1967. En gammel Historie fra dansk til frisisk (Moringermål) oversat af Bende Bendsen (1860). København.

Gonnsen, August. 1993. Soowen frasche tääle. Milton, Canada: Alpha Associates. 


\section{US WURK LXX (2021), p. 83}

Gonnsen, August. o. J. Lene, Bäne än Hansiine. En spal önj tra apträäse. Hs. Nordfriesische Wörterbuchstelle der Christian-Albrechts-Universität zu Kiel.

Hamsen, Mattgetrehn Jans. 1929. Fraschen Kalender. (o.J.): Drückt öntj ejgenen Verlag.

Heitmann, Elise. 1958. Frasche tääle üt Nord-, Ååst- än Weestfraschlönj; üt Tjüschlönj än Dånmark. o.O.

Heitmann, Elise. 1973. Min lönj! (eefter Nis Petersen). Nordfriesland 7, 73.

Hoekstra, Jarich. 1993. Ig-tiidwurden en g-tiidwurden. Us Wurk: Tydskrift foar Frisistyk 4, 21-68

Hoekstra, Jarich. 1998. Fryske wurdfoarming. Ljouwert: Fryske Akademy.

Hoekstra, Jarich. 2001. Comparative Aspects of Frisian Morphology. In: Horst H. Munske et al. (Hrsg.), Handbuch des Friesischen / Handbook of Frisian Studies. Tübingen: Niemeyer, 775-786.

Hoekstra, Jarich (Hrsg.). 2009. Die Weimarer Konversationen. Onnerreesingä aaf Freesk (1760), auerseetet voon Boy Jacobsen. Kiel: Friesische Philologie / Nordfriesische Wörterbuchstelle.

Hoekstra, Jarich. 2015. Mooringer Grammatik. Kiel. Online verfügbar unter https://www.frisistik-thesaurus.uni-kiel.de/de/dialekte-1/bkingharderfriesisch/grammatiken/mooring-grammatik (letzter Zugriff am 10.07. 2020).

Holthausen, Ferdinand. 1938. Nordfriesische Studien V. 2. Ein nordfries. Glückwunsch von 1746. Beiträge zur Geschichte der deutschen Sprache und Literatur 62, 155-157.

Ingwersen, Katharina \& Albrecht Johannsen (Hrsg.). 1926. Frasch Leseböck. Lesebuch für die friesischen Festlandsharden zum Schulgebrauch und fürs Haus. o. O.: Nordfriesiche Rundschau G.m.b.H.

Jacobsen, Boy. 1743. Friesisches Wörterbuch. Göttingen: Ms. der Niedersächsischen Staats- und Universitätsbibliothek in Göttingen, Ms. philol. 244.

Jacobsen, Boy. ca. 1745. Krästian Aernst Stienbecks tiösk Waardä-Bauck, aunt freesk auerseetet voon BJ. Photokopie der Nordfriesischen Wörterbuchstelle der Universität Kiel.

Jannsen, Herrlich. 1956-1958. Häl en junk - Sö schrif en toocht en Foomen. Südtondern Tageblatt Nr. 265 (10.11.1956) - Nr. 253 (30.10.1958).

Jannsen, Thusnelda. 1921. Alerhand Frasches to Jüll 1921 for gaue Frasche. Niebüll-Deezbüll.

Jörgensen, V. Tams. 1968, 1972² , 1976³ $1978^{4}$, Reprint 1986. Kort Spräkeliir foon dåt Mooringer Frasch. Bräist: Nordfriisk Instituut. 


\section{US WURK LXX (2021), p. 84}

Köbernik, Grete Marie. 2014. Unregelmäßige Verben im Friesischen: Vier Dialekte im Vergleich. Masterarbeit im Zwei-Fächer-Masterstudiengang, Fach Friesische Philologie der Philosophischen Fakultät der ChristianAlbrechts-Universität zu Kiel.

Kramer, Pyt. 1982. Kute Seelter Sproakleere. Kurze Grammatik des Saterfriesischen. Rhauderfehn: Ostendorp Verlag.

Lyngby, K. J. 1858. Om Nordfrisisk i Bøkking og Hvidding Herreder (Nibøl og Klangsbøl Sogne). København: Eibe / Leipzig: Lorck.

Nissen, Moritz Momme. 1873-1883. De Frèske Findling, dat sen sprèkkwurde önt Karhirdinge, Mourange, Withinge, Sellange, Amringe, Hatstinge, Breklinge, Westfrèske an Engelske Reth. Stedesand: Selbstverlag des Verfassers.

Nommensen, Ingwer. 1985. En fïrngstün Hamlet. Nordfriesland 19, 51-57.

Nommensen, Ingwer. 1988. Di suurte hün foont Risem Måår. En kameedistuk önj seeks bile. Nordfriesland 22, 15-20

Petersen, Adeline. 2007. Friesischer Sprachkurs. Mooringer Frasch. Bräist/ Bredstedt: Nordfriisk Instituut.

Petersen, Adeline \& Ingo Laabs. 2008. Friesischer Sprachkurs. Mooringer Frasch II. Bräist/Bredstedt: Nordfriisk Instituut.

Petersen, Erk. 2017. Deer driif en heef foon't sööden jurt. En ütwool foon toochte, tääle än dächte. Herausgegeben von Ingo Laabs. Kiel: Friesische Philologie / Nordfriesische Wörterbuchstelle.

Rask, Rasmus. 1825. Frisisk sproglaere, udarbejdet efter samme plan som den islandske og angelsaksiske. København: Beeken.

Sjölin, Bo. 1986. Frasch for önjfångere. Programmierter Lehrgang des Mooringer Friesisch. Nordfriesische Wörterbuchstelle der ChristianAlbrechts-Universität zu Kiel.

Sjölin, Bo, Alastair G.H. Walker \& Ommo Wilts. 1988. Frasch uurdebök. Wörterbuch der Mooringer Mundart. Neumünster: Wachholtz.

Tångeberg, Marie. 1993². Eemil foon Lönebärj (Astrid Lindgren). 2. Ferbääderde aplååge. Risem/Lunham: Foriining for Nationale Friiske.

Tångeberg, Marie. 1992. Föögle önj Nordfraschlönj. Risem-Lunham/ Risum-Lindholm: Foriining for nationaale Friiske.

Tångeberg, Marie. 1995. Söl önj bile an tääle. Bräist/Bredstedt: Nordfriisk Instituut.

Tångeberg, Marie. 2020. Dåt loschiir-hüs bai e Thames. Bräist/Bredstedt: Friisk Foriining.

Tiersma, Pieter Meijes. $1999^{2}$. Frisian reference grammar. Ljouwert: Fryske Akademy. 


\section{US WURK LXX (2021), p. 85}

Walker, Alastair G.H. 1990. Frisian. In: Charles V.J. Russ (Hrsg.), The Dialects of Modern German. A Linguistic Survey. London: Routledge, 1-30.

Walker, Alastair G.H. \& Ommo Wilts. 2001. Die nordfriesischen Mundarten. In: Horst H. Munske et al. (Hrsg.), Handbuch des Friesischen / Handbook of Frisian Studies. Tübingen: Niemeyer, 284-304.

Wilts, Ommo. 1977. Drei friesische »Königsgedichte« von Carsten Johannsen (1798-1856). Nordfriesisches Jahrbuch 13, 205-213.

Wilts, Ommo. 1995. Friesische Formenlehre in Tabellen V. Bökingharde. Husum: Matthiesen. 